\title{
THE CORONA FACTORIZATION PROPERTY, STABILITY, AND THE CUNTZ SEMIGROUP OF A $C^{*}$-ALGEBRA
}

\author{
EDUARD ORTEGA, FRANCESC PERERA, AND MIKAEL RØRDAM
}

\begin{abstract}
The Corona Factorization Property, originally invented to study extensions of $C^{*}$-algebras, conveys essential information about the intrinsic structure of the $C^{*}$-algebra. We show that the Corona Factorization Property of a $\sigma$-unital $C^{*}$-algebra is completely captured by its Cuntz semigroup (of equivalence classes of positive elements in the stabilization of $A$ ). The corresponding condition in the Cuntz semigroup is a very weak comparability property termed the Corona Factorization Property for semigroups. Using this result one can for example show that all unital $C^{*}$-algebras with finite decomposition rank have the Corona Factorization Property.

Applying similar techniques we study the related question of when $C^{*}$-algebras are stable. We give an intrinsic characterization, that we term property (S), of $C^{*}$ algebras that have no non-zero unital quotients and no non-zero bounded 2-quasitraces. We then show that property ( $\mathrm{S}$ ) is equivalent to stability provided that the Cuntz semigroup of the $C^{*}$-algebra satisfies another (also very weak) comparability property, that we call the $\omega$-comparison property.
\end{abstract}

\section{INTRODUCTION}

The Corona Factorization Property was defined and studied by Kucerovsky and Ng in [12] building on work by Elliott and Kucerovsky, [6], in which purely large $C^{*}$-algebras were studied. Both concepts relate to the theory of extensions and in particular to the important question on when extensions are automatically absorbing.

A $C^{*}$-algebra satisfies the Corona Factorization Property if every full projection in the multiplier algebra of its stabilization is properly infinite (and hence equivalent to the unit). The existence of non-properly infinite full projections in the multiplier algebra of a stable $C^{*}$-algebra was noted (implicitly) in [19], and more explicitly in [20], in connection with the construction of non-stable $C^{*}$-algebras that become stable when being tensored with a matrix algebra. The existence of finite full projections in the multiplier algebra of a stable $C^{*}$-algebra was also essential in the construction in [21] of a simple $C^{*}$-algebra with a finite and an infinite projection. In the language of Kucerovsky and $\mathrm{Ng}$ it is shown in 21 that the $C^{*}$-algebra $C\left(\prod_{n=1}^{\infty} S^{2}\right)$ does not have the Corona Factorization Property.

Date: October 24, 2018.

2000 Mathematics Subject Classification. Primary 16D70, 46L35; Secondary 06A12, 06F05, 46L80.

Key words and phrases. $C^{*}$-algebras, Corona Factorization, Cuntz semigroup, decomposition rank. 
Zhang proved a (partial) converse of these results, that a simple $C^{*}$-algebra of real rank zero with the Corona Factorization Property is either stably finite or purely infinite.

It thus appears that failure to have the Corona Factorization Property is an "infinite dimensional" property, and conversely that all $C^{*}$-algebras with "finite dimensional behavior" should have the Corona Factorization Property. (By finite and infinite dimensinality we are, of course, not referring to the vector space dimension of the $C^{*}$-algebra, but rather to its non-commutative dimension - perhaps best defined through Kirchberg and Winter's notion of decomposition rank.) Pimsner, Popa, and Voiculescu studied in [15] extensions of $C(X) \otimes \mathcal{K}$, where $X$ is a finite-dimensional compact metric space, and developed an $\operatorname{Ext}(X,-)$ theory. It follows in particular from their work that $C(X) \otimes \mathcal{K}$ has the Corona Factorization Property when $X$ has finite dimension. The assumption that $X$ is finite dimensional is crucial.

Using Kirchberg and Winter notion of decomposition rank, [11], mentioned above, Kucerovsky and $\mathrm{Ng}$, 13], studied extensions of type I $C^{*}$-algebras with finite decomposition.

In this paper we show that a $\sigma$-unital $C^{*}$-algebra (simple or not) satisfies the Corona Factorization Property if, and only if, its Cuntz semigroup satisfies a very weak comparison property that we call the Corona Factorization Property for semigroups (also considered in [14]). We also introduce stronger notions of comparison for ordered abelian semigroups, some of which are verified for the Cuntz semigroup of a unital $C^{*}$-algebra with finite decomposition rank, and so the Corona Factorization Property also holds for these algebras.

This parallels the property that the authors introduced and examined in the article [14]. There it was shown, using entirely different techniques than those employed here, that a $\sigma$-unital $C^{*}$-algebra of real rank zero has the Corona Factorization Property if and only if its monoid $V(A)$ of Murray-von Neumann equivalence classes of projections in the stabilization of a $C^{*}$-algebra $A$ has the Corona Factorization Property (for monoids).

In outline the paper is as follows. In Section 2, we define and consider various comparability properties for ordered abelian semigroups, including $n$-comparison and $\omega$ comparison (and their weak counterparts), and the Corona Factorization Property for semigroups. These properties can be viewed as weakened forms of the almost unperforation property for semigroups (considered in [22]). In fact, an ordered abelian semigroup has the 0-comparison property if and only if it is almost unperforated. It follows from a result of Toms and Winter, [23], that the Cuntz semigroup of any unital simple $C^{*}$-algebra with decomposition rank $n$ has the $n$-comparison property (and hence also $\omega$-comparison and the Corona Factorization Property). It was this result by Toms and Winter that led us to consider $n$-comparison.

In Section 3 we establish (using the above mentioned result of Toms and Winter) that the Cuntz semigroup of (non-simple) unital $C^{*}$-algebras with finite decomposition rank has the weak $n$-comparison property, and hence also the Corona Factorization property. After the first version of this paper was posted Leonel Robert proved that the Cuntz 
semigroup of any $C^{*}$-algebra with nuclear dimension at most $n$ (respectively, $\omega$ ) has $n$-comparison (respectively, $\omega$-comparison), [16].

In Section 4 we consider an intrinsic property (that we call property $(\mathrm{S})$ ) of a $C^{*}$ algebra defined in terms of Cuntz' comparison theory for $C^{*}$-algebras, and we show that it is equivalent to the absence of unital quotients and bounded 2-quasitraces. It is further shown that property $(\mathrm{S})$ is equivalent to stability of a $C^{*}$-algebra if its Cuntz semigroup has the $\omega$-comparison property, thus generalizing a result from [8].

In Section 5 we prove our main result, that a $\sigma$-unital $C^{*}$-algebra has the Corona Factorization Property if and only if its Cuntz semigroup satisfies the comparability condition called the Corona Factorization Property (for semigroups), and that every ideal in a $\sigma$-unital $C^{*}$-algebra has the Corona Factorization Property if and only if its Cuntz semigroup satisfies the strong Corona Factorization Property (for semigroups).

\section{Comparability in ORDERED ABElian SEMigroups}

In this section we shall discuss a number of comparability properties of ordered abelian semigroups.

Consider an ordered abelian semigroup $(W,+, \leq)$ that we here and in what is to follow tacitly shall assume to be positive, i.e., $x \leq x+z$ for all $x, z \in W$. We do not assume that the order is the algebraic one, given by $x \leq y$ if and only if $y=x+z$ for some $z$ in $W$.

A state on $W$ normalized at $x \in W$ is an additive order preserving map from $W$ into $\mathbb{R}^{+} \cup\{\infty\}$ that maps $x$ to 1 . The set of all states normalized at $x$ is denoted by $S(W, x)$. Given two elements $x, y \in W$, one writes $x \propto y$ if there exists $n \in \mathbb{N}$ such that $x \leq n y$.

The result below has appeared already in several versions in the literature, perhaps first as the extension result of Goodearl and Handelman in [7, Lemma 4.1]. We wish to emphasize the following formulation that will be essential for our paper.

Proposition 2.1. Let $(W,+, \leq)$ be an ordered abelian semigroup, and let $x, y \in W$. Then the following statements are equivalent:

(i) There exists $k \in \mathbb{N}$ such that $(k+1) x \leq k y$.

(ii) There exists $k_{0} \in \mathbb{N}$ such that $(k+1) x \leq k y$ for every $k \geq k_{0}$.

(iii) $x \propto y$ and $f(x)<f(y)$ for every $f \in S(W, y)$.

If $S(W, y)$ is empty, then statement (iii) above reduces to the statement $x \propto y$.

Proof. (iii) $\Rightarrow$ (i). Assume first that $y$ is an order unit for $W$ and that (iii) holds. Use compactness of $S(W, y)$ to find natural numbers $m>m^{\prime}$ such that $f(m x)<f\left(m^{\prime} y\right)$ for all $f \in S(W, y)$. By the results of Goodearl and Handelman in [7] there exist a natural number $n$ and an element $z$ in $W$ such that $n m x+z \leq n m^{\prime} y+z$. From this inequality we get $r n m x+z \leq r n m^{\prime} y+z$ for all natural numbers $r$. Now, $z \leq p y$ for some natural number $p$ (because $y$ was assumed to be an order unit). This gives us $r n m x \leq\left(r n m^{\prime}+p\right) y$ for all natural numbers $r$. Choose $r$ large enough so that $r n m>r n m^{\prime}+p$ and put $k=r n m^{\prime}+p$. Then $(k+1) x \leq r n m x \leq k y$. 
If $y$ is not an order unit, then consider the order ideal $W^{\prime}$ in $W$ generated by $y$ (i.e., $W^{\prime}$ consists of all elements $w$ such that $\left.w \propto y\right)$. Then $y$ is an order unit in $W^{\prime}$ and $x$ belongs to $W^{\prime}$. Moreover, every state in $S(W, y)$ restricts to a state in $S\left(W^{\prime}, y\right)$; and every state in $S\left(W^{\prime}, y\right)$ extends to a state in $S(W, y)$ by letting it attain the value $\infty$ in $W \backslash W^{\prime}$. In this way we can reduce the general case to the case dealt with above where $y$ is an order unit.

(ii) $\Rightarrow$ (iii). This is contained in the proof of [22, Proposition 3.2], but here it comes again: First, if $(k+1) x \leq k y$, then $x \leq k y$, so $x \propto y$. Second, $(k+1) x \leq k y$ implies that $f(x) \leq k(k+1)^{-1}<1=f(y)$ for every $f \in S(W, y)$.

(i) $\Rightarrow$ (ii). Suppose $(m+1) x \leq m y$ for some positive integer $m$. Put $k_{0}=(m+1) m$. For each $k \geq k_{0}$ write $k=(m+1) r+s$, where $r$ and $s$ are non-negative integers with $s \leq m$. Note that necessarily $r \geq m$. Therefore

$$
(k+1) x=(m+1) r x+(s+1) x \leq(m+1) r x+(m+1) x \leq m r y+m y \leq k y .
$$

Definition 2.2. Given $x, y$ in an ordered abelian semigroup $W$. Then we say that $x$ is stably dominated by $y$, written $x<_{s} y$, if the equivalent conditions (i)-(iii) in Proposition 2.1 hold.

Remark 2.3. Notice that the relation $<_{s}$ is transitive. Indeed, let $x, y, z \in W$ be such that $x<_{s} y$ and $y<_{s} z$. Then by Proposition 2.1 (ii) there exists $k \in \mathbb{N}$ such that $(k+1) x \leq k y$ and $(k+1) y \leq k z$. But then $(k+1) x \leq k y \leq(k+1) y \leq k z$, so $x<_{s} z$.

Remark 2.4. The notion of almost unperforation from [22, Definition 3.3] can, in terms of stable domination, be rephrased as follows. An ordered abelian semigroup $W$ is almost unperforated if and only if for all $x, y$ in $W, x<_{s} y$ implies $x \leq y$.

Let us briefly remind the reader about the ordered Cuntz semigroup(s) $W(A)$ and $\mathrm{Cu}(A)$ associated to a $C^{*}$-algebra $A$ introduced by Cuntz in [5]. The (uncompleted) Cuntz semigroup $W(A)$ arises as $M_{\infty}(A)^{+} / \approx$, where $M_{\infty}(A)^{+}$denote the positive elements in the disjoint union $\bigcup_{n=1}^{\infty} M_{n}(A)$, and where $\approx$ is Cuntz equivalence: For $a \in M_{n}(A)^{+}$ and $b \in M_{m}(A)^{+}$write $a \precsim b$ if $x_{k}^{*} b x_{k} \rightarrow a$ for some sequence $\left\{x_{k}\right\}$ in $M_{m, n}(A)$, and write $a \approx b$ if $a \precsim b$ and $b \precsim a$. Equip $W(A)$ with addition arising from direct sum $a \oplus b=\operatorname{diag}(a, b) \in M_{n+m}(A)$, and with the order induced by $\precsim$. Let $\langle a\rangle \in W(A)$ denote the equivalence class containing $a$.

In [4], Coward, Elliott, and Ivanescu introduced a modified version of the Cuntz semigroup, $\mathrm{Cu}(A)$. They use suitable equivalence classes of countably generated Hilbert modules (which, in the case of stable rank one, amount to isomorphism) to obtain an ordered abelian semigroup, which in certain good situations can be viewed as the completion of $W(A)$, see [1], and which turns out to be order-isomorphic to $W(A \otimes$ $\mathcal{K})$. Unlike the functor $A \mapsto W(A)$, the new Cuntz semigroup functor $A \mapsto \mathrm{Cu}(A)$ is continuous; and $\mathrm{Cu}(A)$ is closed under suprema of increasing sequences. 
Coward, Elliott, and Ivanescu also formalize the notion of compact containment in the Cuntz semigroup to arbitrary ordered abelian semigroups $W$ that admit suprema of increasing sequences as follows: Given two elements $x, y$ in $W, x$ is compactly contained in $y$, sometimes colloquially referred to as $x$ is way below $y$, and denoted by $x \ll y$, if whenever $\left\{y_{n}\right\}$ is an increasing sequence with supremum greater than or equal to $y$, eventually $x \leq y_{n}$.

A proto-example of compact containment in the Cuntz semigroup is $\left\langle(a-\varepsilon)_{+}\right\rangle \ll\langle a\rangle$ that holds in $\mathrm{Cu}(A)$ for all $C^{*}$-algebras $A$, for all positive elements $a \in A \otimes \mathcal{K}$, and for all $\varepsilon>0$.

Definition 2.5 (Complete ordered abelian semigroups). An ordered abelian semigroup $(W,+, \leq)$ is said to be complete if every increasing sequence in $W$ admits a supremum; and if for every pair of elements $x, y \in W$ the following holds: $x \leq y$ if and only if $x^{\prime} \leq y$ for all $x^{\prime} \in W$ with $x^{\prime} \ll x$.

The Cuntz semigroup $\mathrm{Cu}(A)$ is complete and in fact belongs to the category $\mathbf{C u}$, defined in [4], which is the appropriate frame in which to study this object. For our purposes the weaker notion of completeness will suffice.

Identifying $A$ and matrix algebras over $A$ as hereditary subalgebras of $A \otimes \mathcal{K}$ we can regard $W(A)$ as being a subsemigroup of $\mathrm{Cu}(A)$. Each positive element $a$ in $A$ or in a matrix algebra over $A$ defines a class $\langle a\rangle$ in $\mathrm{Cu}(A)$.

Given $a, b \in A^{+}$write $a \otimes 1_{n}$ to denote the $n$-fold direct sum $a \oplus \cdots \oplus a$. Clearly, $\left\langle a \otimes 1_{n}\right\rangle=n\langle a\rangle$ in $\mathrm{Cu}(A)$. Write $a \prec_{s} b$ if $\langle a\rangle<_{s}\langle b\rangle$. About this relation we have the lemma below, which is similar to [18, Proposition 2.4].

Lemma 2.6. Let $a$ and $b$ be positive elements in $a C^{*}$-algebra $A$ and suppose that $a \prec_{s} b$. Then, for each $\varepsilon>0$ there exists $\delta>0$ such that $(a-\varepsilon)_{+} \prec_{s}(b-\delta)_{+}$.

Proof. For $c \in A^{+}$note that $\left(\left(c \otimes 1_{k}\right)-\eta\right)_{+}=(c-\eta)_{+} \otimes 1_{k}$.

There exists a positive integer $k$ such that $(k+1)\langle a\rangle \leq k\langle b\rangle$. Hence $a \otimes 1_{k+1} \precsim b \otimes 1_{k}$. Let $\varepsilon>0$. It then follows from [18, Proposition 2.4] that there exists $\delta>0$ such that

$$
(a-\delta)_{+} \otimes 1_{k+1}=\left(a \otimes 1_{k+1}-\delta\right)_{+} \precsim\left(b \otimes 1_{k}-\varepsilon\right)_{+}=(b-\varepsilon)_{+} \otimes 1_{k} .
$$

This shows that $(a-\delta)_{+} \prec_{s}(b-\varepsilon)_{+}$.

Let us note some properties that can be deduced from [18, Proposition 2.4] and Lemma 2.6. If $x, y \in \mathrm{Cu}(A)$, then

(a) $x \leq y$ if and only if $x_{0} \leq y$ for every $x_{0} \in \mathrm{Cu}(A)$ with $x_{0} \ll x$;

(b) if $x \leq y$ and if $x_{0} \in \mathrm{Cu}(A)$ is such that $x_{0} \ll x$, then there is $y_{0} \in \mathrm{Cu}(A)$ with $y_{0} \ll y$ and $x_{0} \leq y_{0}$

(c) if $x<_{s} y$ and if $x_{0} \in \mathrm{Cu}(A)$ is such that $x_{0} \ll x$, then there is $y_{0} \in \mathrm{Cu}(A)$ with $y_{0} \ll y$ and $x_{0}<_{s} y_{0}$.

Our first comparability property, given in Definition 2.8 below, is prompted by a result of Toms and Winter, [23, Lemma 6.1]. Recall that if $\tau$ is a positive trace (or a 2quasitrace) in $A$, then one can associate to it a dimension function $d_{\tau}: \mathrm{Cu}(A) \rightarrow[0, \infty]$ 
given by

$$
d_{\tau}(\langle a\rangle)=\lim _{n \rightarrow \infty} \bar{\tau}\left(a^{1 / n}\right),
$$

where $a$ is a positive element in $A \otimes \mathcal{K}$ and $\bar{\tau}$ is the usual extension of $\tau$ to $A \otimes \mathcal{K}$. The trace property ensures that $d_{\tau}$ is well-defined. We can also view $d_{\tau}$ as being a function on the positive elements in $A \otimes \mathcal{K}$. We shall not distinguish between the two situations.

Proposition 2.7 (Toms and Winter). Let $A$ be a simple, separable, and unital $C^{*}$ algebra of decomposition rank $n<\infty$. Let $a, d_{0}, d_{1}, \ldots, d_{n}$ be positive elements in $A$ such that $d_{\tau}(a)<d_{\tau}\left(d_{j}\right)$ for $j=0,1, \ldots, n$ and for all tracial states $\tau$ on $A$ (where $d_{\tau}$ is the dimension function on $A$ associated with the trace $\tau)$. It follows that

$$
\langle a\rangle \leq\left\langle d_{0}\right\rangle+\left\langle d_{1}\right\rangle+\cdots+\left\langle d_{n}\right\rangle
$$

in the Cuntz semigroup $\mathrm{Cu}(A)$.

Definition 2.8 (The $n$-comparison property). Let $(W,+, \leq)$ be an ordered abelian semigroup and let $n$ be a natural number. Then $W$ is said to have the $n$-comparison property if whenever $x, y_{0}, \ldots, y_{n}$ are elements in $W$ with $x<_{s} y_{j}$ for all $j$, then $x \leq$ $y_{0}+y_{1}+\cdots+y_{n}$.

Note that $W$ has the 0-comparison property if and only if $W$ is almost unperforated, cf. Remark 2.4. Note also, that if $W$ has the $n$-comparison property for some $n$, then it has the $m$-comparison property for all $m \geq n$.

With Definition 2.8 at hand we can rephrase the proposition of Toms and Winter above as follows:

Proposition 2.9. Let $A$ be a simple, separable and unital $C^{*}$-algebra with decomposition rank $n<\infty$. Then $W(A)$ and $\mathrm{Cu}(A)$ have the $n$-comparison property.

Proof. By Lemma 2.10 below it suffices to show this for $W(A)$.

Let $x, y_{0}, \ldots, y_{n} \in W(A)$ be such that $x<_{s} y_{j}$ for every $j=0, \ldots, n$. Upon replacing $A$ by a matrix algebra over $A$ (which does not change the decomposition rank) we may assume that there are positive elements $a, d_{0}, d_{1}, \ldots, d_{n}$ in $A$ such that $x=\langle a\rangle$ and $y_{j}=\left\langle d_{j}\right\rangle$.

We know from Proposition 2.1 that $f(x)<f\left(y_{j}\right)$ for every dimension function $f$ on $A$ normalized at $y_{j}$. As $A$ is simple and unital, every such $f$ is a multiple of a dimension function which is normalized at the unit: $\left\langle 1_{A}\right\rangle$. It follows that $d_{\tau}(a)<d_{\tau}\left(d_{j}\right)$ for every tracial state $\tau$ on $A$ (because $d_{\tau}$ then is a dimension function on $A$ normalized at $1_{A}$ ) Thus, by [23, Lemma 6.1] (which in fact is Proposition 2.7), we get that $a \precsim$ $d_{0} \oplus d_{1} \oplus \cdots \oplus d_{n}$, which in turn implies that $x \leq y_{0}+y_{1}+\cdots+y_{n}$, as desired.

As mentioned in the introduction, Leonel Robert improved this result after the first version of this paper was made public, see [16].

Lemma 2.10. Let $A$ be a $C^{*}$-algebra, and suppose that $W(A)$ has the $n$-comparison property. Then $\mathrm{Cu}(A)$ has the n-comparison property. 
Proof. Let $x, y_{0}, y_{1}, \ldots, y_{n}$ in $\mathrm{Cu}(A)$ be given satisfying $x<_{s} y_{j}$ for all $j$. As $x$ is the supremum of an increasing sequence of elements $\left\{x_{k}\right\}$ from $W(A)$ each satisfying $x_{k} \ll x$ it suffices to show that $x^{\prime} \leq y_{0}+\cdots+y_{n}$ for every $x^{\prime} \in W(A)$ with $x^{\prime} \ll x$. Each $y_{j}$ is likewise the supremum of an increasing sequence $\left\{y_{j}^{(k)}\right\}$ of elements from $W(A)$. Use property (c) above to conclude that there is a natural number $k$ such that $x^{\prime}<_{s} y_{j}^{(k)}$ holds for all $j$. As the inclusion mapping $W(A) \hookrightarrow \mathrm{Cu}(A)$ is an order embedding, the inequality $x^{\prime}<_{s} y_{j}^{(k)}$ also holds in $W(A)$. It follows that $x^{\prime} \leq y_{0}^{(k)}+\cdots+y_{n}^{(k)} \leq y_{0}+\cdots+y_{n}$ as desired.

We proceed to define a comparison property which is weaker than the $n$-comparison property for all $n$.

Definition 2.11 (The $\omega$-comparison property). A complete ordered abelian semigroup $(W,+, \leq)$ is said to have the $\omega$-comparison property if whenever $x^{\prime}, x, y_{0}, y_{1}, y_{2}, \ldots$ are elements in $W$ such that $x<_{s} y_{j}$ for all $j$ and $x^{\prime} \ll x$, then $x^{\prime} \leq y_{0}+y_{1}+\cdots+y_{n}$ for some $n$ (that may depend on the element $x^{\prime}$ ).

It is clear that if $W$ has the $n$-comparison property for some $n$, then $W$ also has the $\omega$-comparison property.

We shall now (re-)define two even weaker comparison properties for an ordered abelian semigroup, the strong Corona Factorization Property and the Corona Factorization Property. These were also defined (slightly differently) in our paper, [14, written in parallel with this paper. In [14] we were interested in the case of the (discrete) algebraically ordered semigroup, $V(A)$, of Murray-von Neumman equivalence classes of projections. In the present case of the Cuntz semigroup, one has to take into consideration its continuous aspect where the definition below is more appropriate (and it extends the definition given in [14], see Remark 2.16). We shall sometimes refer to the definitions of the Corona Factorization Property in [14] as the discrete versions and the ones given below as the continuous verions.

Definition 2.12 (The strong Corona Factorization Property for semigroups). Let $(W,+, \leq)$ be a complete ordered abelian semigroup. Then $W$ is said to satisfy the strong Corona Factorization Property if given any $x^{\prime}, x$ in $W$, any sequence $\left\{y_{n}\right\}$ in $W$, and any positive integer $m$ satisfying $x^{\prime} \ll x$ and $x \leq m y_{n}$ for all $n$, then there exists a positive integer $k$ such that $x^{\prime} \leq y_{1}+y_{2}+\cdots+y_{k}$.

Fullness, as defined below, was also considered in [14], and again we must extend this definition so that it applies to complete ordered (positive) semigroups.

Definition 2.13 (Full elements and sequences). Let $(W,+, \leq)$ be a complete ordered abelian semigroup.

An element $x$ in $W$ is said to be full if for any $y^{\prime}, y \in W$ with $y^{\prime} \ll y$, one has $y^{\prime} \propto x$.

A sequence $\left\{x_{n}\right\}$ in $W$ is said to be full if it is increasing and for any $y^{\prime}, y \in W$ with $y^{\prime} \ll y$, one has $y^{\prime} \propto x_{n}$ for some (hence all sufficiently large) $n$. 
Every order unit in $W$ is full, but the reverse is not always true. The constant sequence $\left\{x_{n}\right\}$, with $x_{n}=x$ for all $n$, is full if and only if $x$ is full.

Suppose that $A$ is a $C^{*}$-algebra and that $\left\{a_{n}\right\}$ is a sequence of positive elements in $A$. Then $\left\{\left\langle a_{n}\right\rangle\right\}$ is full in $\mathrm{Cu}(A)$ if and only if $a_{1} \precsim a_{2} \precsim a_{3} \precsim \cdots$ and $\left\{a_{n}\right\}$ is full in $A$ (in the sense of $\left\{a_{n}\right\}$ not being contained in a proper closed two-sided ideal in $A$ ).

Definition 2.14 (Corona Factorization Property for semigroups). Let $(W,+, \leq)$ be a complete ordered abelian semigroup. Then $W$ is said to satisfy the Corona Factorization Property if given any full sequence $\left\{x_{n}\right\}$ in $W$, any sequence $\left\{y_{n}\right\}$ in $W$, an element $x^{\prime}$ in $W$, and a positive integer $m$ satisfying $x^{\prime} \ll x_{1}$ and $x_{n} \leq m y_{n}$ for all $n$, then there exists a positive integer $k$ such that $x^{\prime} \leq y_{1}+y_{2}+\cdots+y_{k}$.

Remark 2.15. The content of this remark was suggested to us by Leonel Roberts and George Elliott.

Suppose that $W$ is a complete ordered abelian semigroup that contains a full sequence (this is the case whenever $W=\mathrm{Cu}(A)$ where $A$ is a separable $C^{*}$-algebra). Then, letting $u$ be the supremum of any full sequence in $W$, it follows that the supremum, $p$, of the sequence $\{n u\}$ is the largest element in $W$, i.e., $w \leq p$ for all $w \in W$. In particular, $p$ is properly infinite because $2 p \leq p$. Obviously $p$ is unique with these properties.

Consider the following property (Q) of $W$ : For every $u \in W$ and for every natural number $m$, if $m u=p$, then $u=p$. (Equivalently, one can ask that $u$ be properly infinite if a multiple of $u$ is equal to $p$.) We show below that property (Q) implies the Corona Factorization Property, but we do not know if the two properties are equivalent in general. One could consider the following stronger property (QQ) of an ordered abelian semigroup $W$ : every element $u$ in $W$ a multiple of which is properly infinite is itself properly infinite. Then clearly (QQ) implies (Q). It is shown in [14, Theorem 5.14] that any refinement monoid that satisfies the Corona Factorization Property also satisfies (Q), so the two conditions are equivalent in this case. It is also shown in [14, Theorem 5.14] that any refinement monoid that satisfies the strong Corona Factorization Property will satisfy (QQ) (and hence (Q)). In [3] it is shown that any complete ordered abelian semigroup with $\omega$-comparison satisfies (QQ) (and hence $(\mathrm{Q})$ ).

We proceed to show that $(\mathrm{Q})$ implies the Corona Factorization Property. Indeed, let $\left\{x_{n}\right\}$ be a full sequence in $W$, let $x^{\prime} \in W$ be such that $x^{\prime} \ll x_{1}$, let $\left\{y_{n}\right\}$ be a sequence in $W$ such that $x_{n} \leq m y_{n}$ for all $n$ and for some fixed $m \in \mathbb{N}$. Assume that condition (Q) above holds. We must show that $x^{\prime} \leq y_{1}+\cdots+y_{k}$ for some $k$.

Note first that the supremum of the sequence $\left\{x_{1}+\cdots+x_{n}\right\}$ is $p$. For all $w^{\prime} \ll w$ in $W$ we have $w^{\prime} \leq \ell x_{n}$ for some natural numbers $\ell, n$. Hence $w^{\prime} \leq x_{1}+\cdots+x_{\ell+n-1}$ which again is less than the supremum of the sequence $\left\{x_{1}+\cdots+x_{n}\right\}$. Let $u$ be the supremum of the sequence $\left\{y_{1}+\cdots+y_{n}\right\}$. Then $m u \geq m\left(y_{1}+\cdots+y_{n}\right) \geq x_{1}+\cdots+x_{n}$ for all $n$. Therefore $m u \geq p$, whence $m u=p$. By assumption, $u=p$. In particular we have that $x_{1} \leq u$. By the definition of compact containment (and of $u$ ) we obtain that $x^{\prime} \leq y_{1}+\cdots+y_{k}$ for some $k$. 
It is clear that any semigroup that satisfies the strong Corona Factorization Property also satisfies the Corona Factorization Property. It was shown in [14 that a conical refinement monoid satisfies the strong Corona Factorization Property if and only if every ideal of the monoid satisfies the Corona Factorization Property. It is not clear if this remains true without assuming the refinement property, but we shall show (implicit in Theorem 5.13) that this also holds for semigroups arising as the Cuntz semigroup of a $\sigma$-unital $C^{*}$-algebra. We shall also see, as in the case of $C^{*}$-algebras with real rank zero, that the Corona Factorization Property defined for semigroups matches the corresponding property for the $C^{*}$-algebras (see Section 5).

If $W$ is an algebraically ordered semigroup, then the definitions given above for the (strong) Corona Factorization Property agree with the corresponding ones in [14]. The connection between the two notions is discussed in the remark below.

Remark 2.16. Recall that an interval in an ordered abelian semigroup $V$ is a nonempty subset $I$ of $V$ which is order-hereditary and upwards directed. An interval $I$ is said to be countably generated if there is a sequence $\left\{x_{n}\right\}$ of elements in $I$ (that can be taken to be increasing) such that $I=\left\{x \in V \mid x \leq x_{n}\right.$ for some $\left.n\right\}$.

The addition of two intervals $I$ and $J$ in $V$ is defined to be

$$
I+J=\{x \in V \mid x \leq y+z \text { with } y \in I, z \in J\} .
$$

Denote by $\Lambda(V)$ the ordered abelian semigroup of all intervals in $V$, where the order is given by set inclusion, and denote by $\Lambda_{\sigma}(V)$ the subsemigroup of $\Lambda(V)$ whose elements are the countably generated intervals in $V$. Both semigroups are complete, cf. Definition 2.5: The supremum of an increasing sequence $\left\{I_{n}\right\}$ of intervals is $\cup_{n=1}^{\infty} I_{n}$. Morever, if $I$ is an interval, then $[0, x] \ll I$ for every $x \in I$. It follows immediately from this observation that if $I$ and $J$ are intervals such that $I^{\prime} \subseteq J$ for all $I^{\prime} \ll I$, then $I \subseteq J$.

Now assume that $V$ is algebraically ordered. Then $V$ satisfies the (strong) Corona Factorization Property (the discrete version defined in [14]) if, and only if, $\Lambda_{\sigma}(V)$ satisfies the (strong) Corona Factorization Property (the continuous version defined here). Let us sketch part of the arguments needed. Assume that $V$ has the strong Corona Factorization Property, let $X, Y_{1}, Y_{2}, \ldots$ be elements in $\Lambda_{\sigma}(V)$, and let $m \in \mathbb{N}$ be such that $X \subseteq m Y_{n}$ for all $n$. Let $X^{\prime} \ll X$ be given. Since $X$ is countably generated by a sequence, say $\left\{x_{n}\right\}$, that without loss of generality can be assumed to be increasing, there is $i$ such that $X^{\prime} \subseteq\left[0, x_{i}\right]$. It suffices to check that $\left[0, x_{i}\right] \subseteq Y_{1}+\cdots+Y_{l}$ for some $l$. As $x_{i}$ belongs to $m Y_{n}$ for all $n$, and since each $Y_{n}$ is upwards directed, there is $y_{n} \in Y_{n}$ such that $x_{i} \leq m y_{n}$. By the assumption that $V$ satisfies the strong Corona Factorization Property, this implies that $x_{i} \leq y_{1}+y_{2}+\cdots+y_{n}$ for some $n$. This again implies $X^{\prime} \subseteq Y_{1}+\cdots+Y_{n}$, and thus proves that $\Lambda_{\sigma}(V)$ has the strong Corona Factorization Property.

In [14, the authors proved that a $C^{*}$-algebra with real rank zero has the (strong) Corona Factorization Property if and only if the projection semigroup $V(A)$ has the corresponding property (discrete version) for semigroups. Since $V(A)$ is algebraically 
ordered, it follows that $\Lambda_{\sigma}(V(A))$ satisfies the (strong) Corona Factorization Property (continuous version) if and only if $V(A)$ satisfies the corresponding property (the discrete version). In particular, since for a $C^{*}$-algebra with real rank zero $A$, one has that $\mathrm{Cu}(A)$ is order-isomorphic to $\Lambda_{\sigma}(V(A))$-see [1, Theorem 5.7] —, our observations yield that, within this class, $V(A)$ has the (strong) Corona Factorization Property (discrete version) if and only if $\mathrm{Cu}(A)$ has the corresponding property (continuous version), if and only if $A$ has the (strong) Corona Factorization Property for $C^{*}$-algebras - the latter equivalence follows from the results in [14].

Proposition 2.17. Any complete abelian ordered semigroup, which satisfies the $\omega$ comparison property, also satisfies the Corona Factorization Property.

Proof. Let $W$ be a complete abelian ordered semigroup with the $\omega$-comparison property. Let $\left\{x_{n}\right\}$ be a full sequence in $W$, let $\left\{y_{n}\right\}$ be another sequence in $W$, let $x^{\prime} \in W$, and let $m$ be a positive integer such that $x^{\prime} \ll x_{1}$ and $x_{n} \leq m y_{n}$ for all $n$. For each integer $n \geq 0$ put

$$
z_{n}=y_{n(m+1)+1}+y_{n(m+1)+2}+\cdots+y_{n(m+1)+m+1}
$$

Then

$$
\begin{aligned}
(m+1) x_{1} & \leq x_{n(m+1)+1}+x_{n(m+1)+2}+\cdots+x_{n(m+1)+m+1} \\
& \leq m\left(y_{n(m+1)+1}+y_{n(m+1)+2}+\cdots+y_{n(m+1)+m+1}\right)=m z_{n}
\end{aligned}
$$

whence $x_{1}<_{s} z_{n}$ for all $n$. It follows that $x^{\prime} \leq z_{0}+z_{1}+\cdots+z_{n}$ for some $n$, which entails that $x^{\prime} \leq y_{1}+y_{2}+\cdots+y_{(n+1)(m+1)}$.

We shall finally consider the following notion of $n$-comparison that only involves full elements of the semigroup. (This shall be appropriate for studying the Corona Factorization Property for non-simple $C^{*}$-algebras of finite decomposition rank.)

Definition 2.18 (Weak $n$ - and weak $\omega$-comparison property). Let $(W,+, \leq)$ be an ordered abelian semigroup. Say that $W$ has the weak $n$-comparison property if whenever $y_{0}, y_{1}, \ldots, y_{n}$ are full elements in $W$ and $x \in W$ are such that $x<_{s} y_{i}$ for all $i$, then $x \leq y_{0}+y_{1}+\cdots+y_{n}$.

If $W$ is complete, then we say that $W$ has the weak $\omega$-comparison property if whenever $x, x^{\prime}, y_{0}, y_{1}, \ldots$, are elements in $W$ such that $y_{0}, y_{1}, \ldots$ are full elements, $x^{\prime} \ll x$, and $x<_{s} y_{i}$ for all $i$, then $x^{\prime} \leq y_{0}+y_{1}+\cdots+y_{n}$ for some positive integer $n$.

The weak $n$ - and the weak $\omega$-comparison properties only makes sense for semigroups that contain a full element (if they don't, then they automatically possess this property). It is clear that if $W$ satisfies the weak $n$-comparison property for some $n$, then it satisfies the weak $m$-comparison property for all $m \geq n$ and it satisfies the weak $\omega$-comparison property.

We show below that if $W(A)$ has the weak $n$-comparison property then so does $\mathrm{Cu}(A)$. A new definition and a lemma is required for the proof. 
We say that an element $d$ in a $C^{*}$-algebra $A$ is strictly full if $(d-\varepsilon)_{+}$is full for some $\varepsilon>$ 0 , and hence for all sufficiently small $\varepsilon>0$. Note that any full projection automatically is strictly full. The lemma below characterizes $C^{*}$-algebras whose primitive ideal space is compact (or, equivalently, $C^{*}$-algebras that do not contain proper dense algebraic ideals):

Lemma 2.19. Let $A$ be a $C^{*}$-algebra such that $A \otimes \mathcal{K}$ contains a strictly full positive element. Then every full positive element in $A$ is strictly full.

Proof. View $A$ as a full hereditary sub- $C^{*}$-algebra of its stabilization $A \otimes \mathcal{K}$. Let $a$ be a strictly full positive element in $A \otimes \mathcal{K}$ and choose $\delta>0$ such that $(a-\delta)_{+}$is full in $A \otimes \mathcal{K}$. Let $d$ be an arbitrary full positive element in $A$. For each $\varepsilon>0$ consider the closed two-sided ideal $I_{\varepsilon}$ of $A \otimes \mathcal{K}$ generated by $(d-\varepsilon)_{+}$. The closure of $\bigcup_{\varepsilon>0} I_{\varepsilon}$ is a closed two-sided ideal in $A \otimes \mathcal{K}$ which contains $d$ and hence is equal to $A \otimes \mathcal{K}$. Consequently, $\bigcup_{\varepsilon>0} I_{\varepsilon}$ is a dense (algebraic) ideal in $A \otimes \mathcal{K}$, which therefore contains the Pedersen ideal of $A \otimes \mathcal{K}$, which again contains $(a-\delta)_{+}$. It follows that $(a-\delta)_{+}$ belongs to $I_{\varepsilon}$ for some $\varepsilon>0$, whence $I_{\varepsilon}=A \otimes \mathcal{K}$, which again implies that $(d-\varepsilon)_{+}$is full (in $A \otimes \mathcal{K}$ and hence in $A$ ).

If $A$ is as above, if $x \in \mathrm{Cu}(A)$ is a full element, and if $\left\{x_{k}\right\}$ is an increasing sequence in $\mathrm{Cu}(A)$ with $x=\sup _{k} x_{k}$, then $x_{k}$ is full for all sufficiently large $k$. Indeed, there is a (necessarily strictly full) positive element $a$ in $A \otimes \mathcal{K}$ such that $x=\langle a\rangle$. Find $\varepsilon>0$ such that $(a-\varepsilon)_{+}$is (strictly) full. It follows that $x^{\prime}=\left\langle(a-\varepsilon)_{+}\right\rangle$is full. As $x^{\prime} \ll x$ we have $x^{\prime} \leq y_{k}$ for all large enough $k$, and an element is full if it dominates a full element.

Lemma 2.20. Let $A$ be a $C^{*}$-algebra, and assume that $A \otimes \mathcal{K}$ contains a strictly full element and that $W(A)$ has the weak n-comparison property. Then $\mathrm{Cu}(A)$ has the weak $n$-comparison property.

Proof. We proceed exactly as in the proof of Lemma 2.10, but now have that the $y_{j}$ 's in that proof are full in $\mathrm{Cu}(A)$. It follows from Lemma 2.19 above (and the remarks below it) that the $y_{j}^{(k)}$ 's (from the proof of Lemma 2.10) are full (in $\mathrm{Cu}(A)$ and therefore also in $W(A))$ for all large enough $k$. We therefore obtain the desired conclusion as in the proof of Lemma 2.10.

Proposition 2.21. Any complete abelian ordered semigroup, which satisfies the weak $\omega$-comparison property and which contains a full element that is compactly contained in another (full) element, also satisfies the Corona Factorization Property.

Proof. Let $W$ be a complete abelian ordered semigroup with the weak $\omega$-comparison property. By assumption there are elements $v \ll w$ in $W$ such that $v$ is full.

Let $\left\{x_{k}\right\}$ be a full sequence in $W$, let $\left\{y_{k}\right\}$ be another sequence in $W$, let $x^{\prime} \in W$, and let $m$ be a positive integer such that $x^{\prime} \ll x$ and $x_{k} \leq m y_{k}$ for all $k$. By the definition of a full sequence (applied to $v \ll w$ ) we have that $v \propto x_{k}$ for all large enough $k$. Hence $v \propto y_{k}$ for all large enough $k$, whence $y_{k}$ is full whenever $k$ is large enough. 
Upon removing the first finitely many elements from the sequences $\left\{x_{k}\right\}$ and $\left\{y_{k}\right\}$ we can assume that all $y_{k}$ are full.

Let now $z_{0}, z_{1}, z_{2}, \ldots$ be as in the proof of Proposition 2.17 above. Then $z_{0}, z_{1}, z_{2}, \ldots$ are full and $x_{1}<_{s} z_{j}$ for all $j$. It follows that

$$
x^{\prime} \leq z_{0}+z_{1}+\cdots+z_{n}=y_{1}+y_{2}+\cdots+y_{(n+1)(m+1)}
$$

for some $n$, whence $W$ has the Corona Factorization Property.

In conclusion, we have defined the following comparability properties of a complete ordered abelian semigroup, listed in decreasing strength: 0-comparison (which is the same as being almost unperforated), 1-comparison, 2-comparison, ..., $\omega$-comparison, the strong Corona Factorization Property, and the Corona Factorization Property for semigroups. Moreover, we have defined weak $n$ - and the weak $\omega$-comparison properties. We show below that the comparison properties above are in fact strictly decreasing in strength. An example a complete abelian ordered semigroup that has the strong Corona Factorization Property but not the $\omega$-comparison property is constructed in [3] . (That the strong Corona Factorization Property is strictly stronger than the Corona Factorization Property was already noted in [14].)

Example 2.22. Let $n$ be a positive integer, let $W_{n}$ be the subsemigroup of $\mathbb{Z}^{+}$generated by $\{0, n+1, n+2\}$, and equip $W_{n}$ with the algebraic order. Notice that

$$
(n+1)(n+2)-(n+1)-(n+2)=n^{2}+n-1
$$

is the largest natural number that does not belong to $W_{n}$. Suppose that $x, y_{0}, y_{1}, \ldots, y_{n}$ belong to $W_{n}$, that $x<_{s} y_{j}$ for all $j$, and that $x$ is non-zero. Then all $y_{j}$ 's are non-zero, whence

$$
y_{0}+y_{1}+\cdots+y_{n}-x \geq y_{1}+y_{2}+\cdots+y_{n} \geq n(n+1),
$$

(where the ordering above is the usual one in $\mathbb{Z}$ ), which shows that $x \leq y_{0}+y_{1}+\cdots+y_{n}$ (with respect to the order given on $W_{n}$ ). Hence $W_{n}$ has the $n$-comparison property.

On the other hand, if we take $x=n+1$ and $y_{0}=y_{1}=\cdots=y_{n-1}=n+2$, then $x<_{s} y_{j}$ for all $j$, but

$$
y_{0}+y_{1}+\cdots+y_{n-1}-x=n(n+2)-(n+1)=n^{2}+n-1,
$$

which does not belong to $W_{n}$. Hence $x \not \leq y_{0}+y_{1}+\cdots+y_{n-1}$ in $W_{n}$, whence $W_{n}$ does not have the $(n-1)$-comparison property.

Next, put $W_{\omega}=\bigoplus_{n=1}^{\infty} W_{n}$ (as an ordered abelian semigroup). Then $W_{\omega}$ has the $\omega$-comparison property, but does not have the $n$-comparison property for any finite $n$. Indeed, suppose that $x, y^{0}, y^{1}, \ldots$ in $W_{\omega}$ are such that $x<_{s} y^{j}$ for all $j$. Write $x=\left(x_{1}, x_{2}, \ldots\right)$ and $y^{j}=\left(y_{1}^{j}, y_{2}^{j}, \ldots\right)$, with $x_{k}$ and $y_{k}^{j}$ in $W_{k}$ for all $k$. Then $x_{k}=0$ for all $k$ greater than some $k_{0} \in \mathbb{N}$. Since $W_{k}$ has $k_{0}$-comparison when $k \leq k_{0}$, we have

$$
x_{k} \leq y_{k}^{0}+y_{k}^{1}+\cdots+y_{k}^{k_{0}}
$$

(in $W_{k}$ ) for all $k$, whence $x \leq y^{0}+y^{1}+\cdots+y^{k_{0}}$ (in $W_{\omega}$ ). 
Conversely, given a positive integer $n$, choose $x^{\prime}, y_{0}^{\prime}, y_{1}^{\prime}, \ldots, y_{n}^{\prime}$ in $W_{n+1}$ such that $x^{\prime}<_{s} y_{j}^{\prime}$ for all $j$, and such that $x^{\prime} \not \leq y_{0}^{\prime}+y_{1}^{\prime}+\cdots+y_{n}^{\prime}$. (This is possible because $W_{n+1}$ does not have the $n$-comparison property.) Let $x, y_{0}, y_{1}, \ldots, y_{n}$ in $W_{\omega}$ be the elements whose coordinates in the $(n+1)$ position are, respectively, $x^{\prime}, y_{0}^{\prime}, y_{1}^{\prime}, \ldots, y_{n}^{\prime}$, and whose other coordinates are zero. Then $x<_{s} y_{j}$ for all $j$ while $x \not \leq y_{0}+y_{1}+\cdots+y_{n}$. Hence $W_{\omega}$ does not have the $n$-comparison property.

\section{The Corona Factorization Property for $C^{*}$-Algebras with finite DECOMPOSITION RANK}

We have already mentioned the result, [23, Lemma 6.1], of Toms and Winter which implies that the Cuntz semigroup of a simple unital separable $C^{*}$-algebra has $n$-comparison property. We wish to extend this result to the non-simple case, and state for this purpose a lemma whose proof actually is contained in the proof of [23, Lemma 6.1] (follow that proof from Equation (10) to its end) and therefore is omitted.

Lemma 3.1 (Toms and Winter). Let $A$ be a separable $C^{*}$-algebra with finite decomposition rank $n$. Suppose that $a, d_{0}, \ldots, d_{n} \in A^{+}$and $\alpha>0$ satisfy

$$
\forall \tau \in T(A): \quad d_{\tau}(a)<d_{\tau}\left(d_{i}\right)-\alpha,
$$

where $d_{\tau}$ is the dimension function associated to $\tau$ as in (1). Then $a \precsim d_{0} \oplus d_{1} \oplus \cdots \oplus d_{n}$.

Proposition 3.2. Let $A$ be a separable, unital $C^{*}$-algebra with decomposition rank $n<$ $\infty$. Then $W(A)$ and $\mathrm{Cu}(A)$ have the weak $n$-comparison property.

Proof. By Lemma 2.20 it suffices to prove the proposition for $W(A)$.

Let $x, y_{0}, \ldots, y_{n} \in W(A)$ with $y_{i}$ full and $x<_{s} y_{i}$ be given for every $i$. Then, by Proposition 2.1, there exists $k$ such that $(k+1) x \leq k y_{i}$ for all $i$.

As $y_{0}, \ldots, y_{n}$ are full, there is a natural number $N$ such that $\left\langle 1_{A}\right\rangle \leq N y_{i}$ for all $i$. Choose $0<\alpha^{\prime}<(k+1)^{-1}$ and let $\alpha=\alpha^{\prime} / N$. Let now $f \in S\left(W(A),\left\langle 1_{A}\right\rangle\right)$. We then have that $1 \leq N f\left(y_{i}\right)$ for all $i$, whence $\alpha<f\left(y_{i}\right) /(k+1)$. Therefore:

$$
f(x) \leq \frac{k}{k+1} f\left(y_{i}\right)=f\left(y_{i}\right)-\frac{f\left(y_{i}\right)}{k+1}<f\left(y_{i}\right)-\alpha
$$

for all $i$.

In particular, $d_{\tau}(x)<d_{\tau}\left(y_{i}\right)-\alpha$ for all $i$ and for every tracial state $\tau$ on $A$

Finite decomposition rank passes to matrices, so upon replacing $A$ with a matrix algebra over $A$, we can suppose that there exist positive elements $a$ and $d_{0}, d_{1}, \ldots, d_{n}$ in $A$, with $d_{i}$ full, such that $x=\langle a\rangle$ and $y_{i}=\left\langle d_{i}\right\rangle$ for all $i$. Then $d_{\tau}(a)<d_{\tau}\left(d_{i}\right)-\alpha$ for all $i$ and for all tracial states $\tau$ on $A$. Lemma 3.1 then implies that $a \precsim d_{0} \oplus d_{1} \oplus \cdots \oplus d_{n}$, which again implies that $x \leq y_{0}+y_{1}+\cdots+y_{n}$ as desired.

Combining Proposition 2.21 and Proposition 3.2 we get:

Corollary 3.3. Let $A$ be a separable, unital $C^{*}$-algebra with finite decomposition rank. Then $\mathrm{Cu}(A)$ has the Corona Factorization Property. 


\section{Stability of $C^{*}$-Algebras}

We show in this section that a $C^{*}$-algebra whose Cuntz semigroup has the $\omega$-comparison property is stable if and only if it has no unital quotient and no bounded 2-quasitrace. We introduce a property $(\mathrm{S})$ of a $C^{*}$-algebra that we show is equivalent to having no non-zero unital quotients and no bounded 2-quasitraces.

It was shown in [9] that a separable $C^{*}$-algebra $A$ is stable if and only if to every $a \in F(A)$ there exists $b \in A^{+}$such that $a \perp b$ and $a \precsim b$. (The set $F(A)$ consists of all positive elements $a$ in $A$ for which $a=a e$ for some positive element $e$ in $A$ (that can be taken to be a contraction).) We shall here consider a weaker version of this condition, where we replace the relation $a \precsim b$ with the relation $a \prec_{s} b$ considered in Section 2 .

Definition 4.1. A $C^{*}$-algebra $A$ is said to have property $(S)$ if for every $a \in F(A)$ there exists $b \in A^{+}$such that $a \perp b$ and $a \prec_{s} b$.

It follows immediately from the definition, the results from [9] quoted above, and from Remark 2.4, that if $A$ is a separable $C^{*}$-algebra for which $\mathrm{Cu}(A)$ is almost unperforated, then $A$ has property $(\mathrm{S})$ if and only if $A$ is stable. It is easy to see that every stable $C^{*}$-algebra has property $(\mathrm{S})$.

Lemma 4.2. Let $A$ be a separable $C^{*}$-algebra with property $(S)$. Then $A$ has no non-zero unital quotients.

Proof. Let $I$ be an ideal of $A$ such that $A / I$ is unital. Let $e+I$ be the unit of $A / I$, with $e \in A^{+}$. Upon replacing $e$ with $g(e)$, where $g: \mathbb{R}^{+} \rightarrow[0,1]$ is a continuous function which vanishes on, say $[0,1 / 2]$, and with $g(1)=1$, we can assume that $e \in F(A)$. By the assumption that $A$ has property (S) there exists $b \in A^{+}$such that $e \perp b$ and $e \prec_{s} b$. Now, $0=e b+I=b+I$, so $b$ belongs to $I$. The relation $e \prec_{s} b$ implies that $e$ belongs to the closed two-sided ideal generated by $b$, and hence to $I$. Thus, $e+I=0$ and $A / I=0$.

Lemma 4.3. Let $A$ be a separable $C^{*}$-algebra with property $(S)$. Then given any $a \in$ $F(A)$ there exists $b \in F(A)$ such that

$$
a \perp b, \quad a \prec_{s} b, \quad a+b \in F(A) .
$$

If, moreover, $A \otimes \mathcal{K}$ is assumed to contain a strictly full positive element, then $b$ above can be chosen to be strictly full in $A$.

Proof. Let $a \in F(A)$, and choose $d$ in $A^{+}$with $d a=a d=a$. Let $g: \mathbb{R}^{+} \rightarrow[0,1]$ be a continuous function which is zero on $[0,1 / 2]$ and with $g(1)=1$, and put $e=g(d)$. Then

$$
e \in F(A), \quad e a=a e=a, \quad a \precsim(e-1 / 2)_{+}, \quad\|e\|=1 .
$$

Since $A$ has property (S) there exists $b_{0} \in A^{+}$such that $e \perp b_{0}$ and $e \prec_{s} b_{0}$. It follows from Lemma 2.6 that there exists $\delta>0$ such that $(e-1 / 2)_{+} \prec_{s}\left(b_{0}-\delta\right)_{+}$. Put 
$b=\left(b_{0}-\delta\right)_{+} \in F(A)$, and set $f=h\left(b_{0}\right)$ where $h: \mathbb{R}^{+} \rightarrow[0,1]$ is a continuous function such that $h(0)=0$ and $h(t)=1$ for $t \geq \delta$. Then $a \perp b, a \prec_{s} b$, and

$$
(e+f)(a+b)=e a+f b=a+b .
$$

The latter shows that $a+b$ belongs to $F(A)$.

Assume now that $A \otimes \mathcal{K}$ contains a strictly full positive element. We show how to modify the proof above so that $b$ becomes strictly full. Let $B$ be the hereditary sub$C^{*}$-algebra of $A$ consisting of all elements which are orthogonal to $e$. Then $B$ is full in $A$. Indeed, because $e$ belongs to $F(A)$ there exists a positive element $e^{\prime}$ in $A$ such that $e^{\prime} e=e e^{\prime}=e$. Let $I$ be the closed two-sided ideal in $A$ generated by $B$, and assume, to reach a contradiction, that $I$ were proper. Then $e^{\prime}+I$ would be a unit for $A / I$, thus contradicting Lemma 4.2.

It follows from Brown's theorem that $B \otimes \mathcal{K}$ is isomorphic to $A \otimes \mathcal{K}$, and so $B \otimes \mathcal{K}$ contains a strictly full positive element. Hence, by Lemma 2.19, any full element in $B$ is strictly full. Upon replacing the element $b_{0}$ from above with the sum of $b_{0}$ and a positive strictly full element in $B$ we can assume that $b_{0}$ is strictly full. It follows that $b=\left(b_{0}-\delta\right)_{+}$is full (and hence strictly full) if $\delta>0$ is chosen sufficiently small.

Lemma 4.4. Let $A$ be a separable $C^{*}$-algebra with property $(S)$. Then for every a $\in$ $F(A)$ there is a sequence $b_{0}, b_{1}, b_{2}, \ldots$ of elements in $F(A)$ such that the elements $a, b_{0}, b_{1}, b_{2}, \ldots$ are pairwise orthogonal, $a+b_{0}+b_{1}+\cdots+b_{n}$ belongs to $F(A)$ for all $n$, and such that $a \prec_{s} b_{0} \prec_{s} b_{1} \prec_{s} \cdots$.

If, moreover, $A \otimes \mathcal{K}$ is assumed to contain a strictly full positive element, then $b_{0}, b_{1}, b_{2}, \ldots$ above can be chosen to be strictly full in $A$.

Proof. The existence of $b_{0}$ such that $a \perp b_{0}, a \prec_{s} b_{0}$, and $a+b_{0}$ belongs to $F(A)$ follows from Lemma 4.3. Suppose that $n \geq 0$ and that $b_{0}, b_{1}, \ldots, b_{n}$ have been found such that $a, b_{0}, b_{1}, \ldots, b_{n}$ are pairwise orthogonal, $a \prec_{s} b_{0} \prec_{s} b_{1} \prec_{s} \cdots \prec_{s} b_{n}$, and $a+b_{0}+b_{1}+\cdots+b_{n}$ belongs to $F(A)$. Then, by Lemma 4.3, there is $b_{n+1}$ in $F(A)$ which is orthogonal to the sum $a+b_{0}+b_{1}+\cdots+b_{n}$ (and hence to each of the summands), such that $a+b_{0}+b_{1}+\cdots+b_{n} \prec_{s} b_{n+1}$ (and hence $b_{n} \prec_{s} b_{n+1}$ ) and such that $a+b_{0}+b_{1}+\cdots+b_{n+1}$ belongs to $F(A)$.

Finally, use Lemma 4.3 to see that each of the positive elements $b_{j}$ above can be chosen to be strictly full if $A \otimes \mathcal{K}$ contains a strictly full positive element.

We will now give an algebraic characterization of property $(\mathrm{S})$ for a $C^{*}$-algebra. The characterization is very similar to, but sharpens, [8, Theorem 3.6]. The reader is referred to [2] for the definition and properties of 2-quasitraces. Let us just here remind the reader than any 2 -quasitrace on an exact $C^{*}$-algebra is a trace, and that the shortcoming of a quasitrace (compared with a trace) is that it only is assumed to be additive on commuting elements. 
Proposition 4.5. Let $A$ be a separable $C^{*}$-algebra. Then $A$ has property $(S)$ if and only if $A$ has no non-zero bounded lower semi-continuous 2-quasitrace and no non-zero unital quotient.

Proof. The "if" part is contained in the proof of [8, Theorem 3.6].

To prove the "only if" part, suppose that $A$ has property (S). By Lemma 4.2, $A$ has no non-zero unital quotients. Suppose, to reach a contradiction, that $\tau$ is a non-zero bounded 2-quasitrace on $A$, and let $d_{\tau}$ be the associated lower semicontinuous dimension function on $W(A)$ (cf., Equation (1)). Since $\tau$ is non-zero there is a positive element $a$ in $A$ such that $d_{\tau}(a)>0$, and since $d_{\tau}$ is lower semicontinuous, $d_{\tau}\left((a-\varepsilon)_{+}\right)>0$ for some $\varepsilon>0$. We can now use Lemma 4.4 to find a sequence $b_{0}=(a-\varepsilon)_{+}, b_{1}, b_{2}, \ldots$ of pairwise orthogonal elements in $F(A)$ such that $b_{0} \prec_{s} b_{1} \prec_{s} b_{2} \prec_{s} \cdots$. By Proposition 2.1 we have $0<d_{\tau}\left(\left\langle b_{0}\right\rangle\right)<d_{\tau}\left(\left\langle b_{1}\right\rangle\right)<d_{\tau}\left(\left\langle b_{2}\right\rangle\right)<\cdots$, and in particular

$$
d_{\tau}\left(\left\langle b_{0}+b_{1}+\cdots+b_{n}\right\rangle\right) \geq(n+1) d_{\tau}\left(\left\langle(a-\varepsilon)_{+}\right\rangle\right) .
$$

On the other hand, one has $d_{\tau}(\langle c\rangle) \leq\|\tau\|$ for all $c$ in $A^{+}$, and so the inequality above is in contradiction with the assumed boundedness of $\tau$.

It is well-known that stability is not a stable property (see [19]). Property (S), however, is a stable property, as easily follows from Proposition 4.5 above:

Corollary 4.6. Let $A$ be a separable $C^{*}$-algebra. Then the following conditions are equivalent:

(i) A has property (S).

(ii) $M_{n}(A)$ has property (S) for some natural number $n$.

(iii) $M_{n}(A)$ has property (S) for all natural numbers $n$.

Proof. By Proposition 4.5 it suffices to check that each of the two properties: having a non-zero unital quotient, and having a non-zero bounded 2-quasitrace, passes to matrix algebras and back again. This is trivial for the first. It is a theorem (see [2]) that 2-quasitraces extend to all matrix algebras (and vice versa).

The result, [8, Theorem 3.6], that we have used extensively in the proof of Proposition 4.5 above, actually says that a separable $C^{*}$-algebra with almost unperforated Cuntz semigroup is stable if and only if it has no non-zero unital quotient and no nonzero bounded 2-quasitrace. Reminding the reader that almost unperforation is the same as the "0-comparison" property, we can extend [8, Theorem 3.6] as follows:

Proposition 4.7. Let $A$ be a separable $C^{*}$-algebra such that $\mathrm{Cu}(A)$ satisfies the $\omega$ comparison property (cf. Definition 2.11). Let $B$ be a hereditary sub-C*-algebra of $A \otimes \mathcal{K}$. Then the following conditions are equivalent:

(i) $B$ is stable

(ii) B has no non-zero unital quotient and no non-zero bounded 2-quasitrace.

(iii) $B$ has property $(S)$. 
Proof. Conditions (ii) and (iii) are equivalent for all separable $C^{*}$-algebras by Proposition 4.5, and (i) clearly implies (ii) (again for all $C^{*}$-algebras) (see eg. [9]).

(iii) $\Rightarrow$ (i). By [9, Proposition 2.2] it is enough to show that for every $a \in B^{+}$ and every $\varepsilon>0$ there exists $b \in B^{+}$such that $(a-\varepsilon)_{+} \precsim b$ and $(a-\varepsilon)_{+} \perp b$. (Indeed, if such an element $b$ exists, then $(a-2 \varepsilon)_{+}=x^{*} b x$ for some $x \in B$; whence $(a-2 \varepsilon)_{+} \sim b^{1 / 2} x x^{*} b^{1 / 2}:=b_{0} \perp(a-2 \varepsilon)_{+}$, and $\left\|a-(a-2 \varepsilon)_{+}\right\| \leq 2 \varepsilon$. $)$

Since $(a-\varepsilon / 2)_{+}$belongs to $F(B)$ we can apply Lemma 4.4 to get a sequence of positive elements $b_{0}, b_{1}, b_{2}, \ldots$ in $F(B)$ such that $(a-\varepsilon / 2)_{+} \prec_{s} b_{0} \prec_{s} b_{1} \prec_{s} b_{2} \prec_{s} \cdots$ and for which $(a-\varepsilon / 2)_{+}, b_{0}, b_{1}, b_{2}, \ldots$ are mutually orthogonal. The corresponding elements

$$
x^{\prime}=\left\langle(a-\varepsilon)_{+}\right\rangle, \quad x=\left\langle(a-\varepsilon / 2)_{+}\right\rangle, \quad y_{j}=\left\langle b_{j}\right\rangle
$$

in $\mathrm{Cu}(A)$ satisfy $x^{\prime} \ll x$ and $x<_{s} y_{j}$ for all $j$. By the assumption that $\mathrm{Cu}(A)$ satisfies the $\omega$-comparison property there is a natural number $n$ such that $x^{\prime} \leq y_{1}+\cdots+y_{n}$. Thus, $(a-\varepsilon)_{+} \precsim b_{0}+\cdots+b_{n}$ (relatively to $A \otimes \mathcal{K}$ and therefore also relatively to the hereditary sub- $C^{*}$-algebra $B$ of $\left.A \otimes \mathcal{K}\right)$ and $(a-\varepsilon)_{+} \perp b_{0}+\cdots+b_{n}$ as desired.

We have the following analog of Proposition 4.7, where the assumption on the comparison property of the Cuntz semigroup is weakened, but where we instead have to assume the existence of a full projection:

Proposition 4.8. Let $A$ be a separable $C^{*}$-algebra such that $\mathrm{Cu}(A)$ satisfies the weak $\omega$-comparison property and such that $A \otimes \mathcal{K}$ contains a full projection. Let $B$ be a full hereditary sub-C $C^{*}$-algebra of $A \otimes \mathcal{K}$. Then the following conditions are equivalent:

(i) $B$ is stable

(ii) B has no non-zero unital quotient and no non-zero bounded 2-quasitrace.

(iii) $B$ has property $(S)$.

Proof. Proceeding as in the proof of Proposition 4.7, we only need to prove (iii) $\Rightarrow$ (i); and to prove that (i) holds it suffices to show that for every $a \in B^{+}$and every $\varepsilon>0$ there exists $b \in B^{+}$such that $(a-\varepsilon)_{+} \precsim b$ and $(a-\varepsilon)_{+} \perp b$.

Since $A \otimes \mathcal{K}$ contains a full projection (which automatically is strictly full), it follows from Lemma 2.19 that all full positive elements in $A \otimes \mathcal{K}$ are strictly full. As $B$ is separable, it contains a full (positive) element, which is then full in $A \otimes \mathcal{K}$ and hence is strictly full in $A \otimes \mathcal{K}$ and therefore also strictly full in $B$. Apply Lemma 4.4 to get full positive elements $b_{0}, b_{1}, b_{2}, \ldots$ in $F(B)$ such that $(a-\varepsilon / 2)_{+} \prec_{s} b_{0} \prec_{s} b_{1} \prec_{s} b_{2} \prec_{s} \ldots$ and such that $(a-\varepsilon / 2)_{+}, b_{0}, b_{1}, b_{2} \ldots$ are mutually orthogonal. Proceed as in the proof of Proposition 4.7 (where it suffices to have the weak $\omega$-comparison property because the elements $y_{j}=\left\langle b_{j}\right\rangle$ are full in $\left.\mathrm{Cu}(A)\right)$ to obtain the desired element $b$.

We end this section by describing when separable $C^{*}$-algebras with finite decomposition rank are stable (under the assumption that their stabilization contains a full projection):

Corollary 4.9. Let $A$ be a separable $C^{*}$-algebra with finite decomposition rank, and assume that $A \otimes \mathcal{K}$ contains a full projection. Then the following conditions are equivalent: 
(i) A is stable.

(ii) A has no non-zero unital quotients and no non-zero bounded positive traces.

(iii) A has property (S).

Proof. Let $p$ be a full projection in $A \otimes \mathcal{K}$ and put $B=p(A \otimes \mathcal{K}) p$. Then $B$ has the same decomposition rank as $A$, say $n$; and $B$ is unital. It follows from Proposition 3.2 that $\mathrm{Cu}(B)$ has weak $n$-comparison and therefore also the weak $\omega$-comparison property. As $A$ is (isomorphic to) a full hereditary sub- $C^{*}$-algebra of $B \otimes \mathcal{K}$ the result now follows from Proposition 4.8. (In (ii) we have used that any 2 -quasitrace on a nuclear $C^{*}$-algebra is a trace.)

\section{The Corona Factorization Property and the Cuntz semigroup}

Recall that a $C^{*}$-algebra $A$ is said to have the Corona Factorization Property if every full projection in the multiplier algebra of $A \otimes \mathcal{K}$ is properly infinite. It was observed by Kucerovsky and $\mathrm{Ng}$ in [12] that the Corona Factorization Property is equivalent to a statement regarding stability of full hereditary sub- $C^{*}$-algebras of the stabilized $C^{*}$-algebra. Our aim here is to characterize the Corona Factorization Property for $C^{*}$ algebras in terms of the comparison property of the Cuntz semigroup of the same name introduced in Section 2. We shall need several lemmas before we can arrive at the main results of this section.

Lemma 5.1. Let $A$ be a $\sigma$-unital $C^{*}$-algebra and suppose that $\left\{e_{k}\right\}$ is an increasing approximate unit for $A$ consisting of positive contractions. Then:

(i) For every positive $a$ in $A$ and for every $\varepsilon>0$ one has $(a-\varepsilon)_{+} \precsim e_{k}$ for all large enough $k$.

(ii) $\left\{\left\langle e_{k}\right\rangle\right\}$ is a full sequence in $\mathrm{Cu}(A)$.

Proof. (i). We have $\left\|a^{1 / 2} e_{k} a^{1 / 2}-a\right\|<\varepsilon$ for $k$ large enough, whence $(a-\varepsilon)_{+} \precsim$ $a^{1 / 2} e_{k} a^{1 / 2} \precsim e_{k}$.

(ii). The sequence $\left\{\left\langle e_{k}\right\rangle\right\}$ is clearly increasing. The fullness property of this sequence follows from (i), from the fact that $\left\{e_{k} \otimes 1_{n}\right\}_{k=1}^{\infty}$ is an approximate unit for $M_{n}(A)$ and that $\bigcup_{n=1}^{\infty} M_{n}(A)$ is dense in $A \otimes \mathcal{K}$.

Recall from Section 4 the definition of the set $F(A)$ of compactly supported elements in a $C^{*}$-algebra $A$. Suppose that $A$ is $\sigma$-unital. Then, to any strictly positive element $c$ in $A$ one can associate the set

$$
F_{c}(A):=\left\{b \in A^{+} \mid g_{\varepsilon}(c) b=b \text { for some } \varepsilon>0\right\},
$$

cf. [9], where $g_{\varepsilon}: \mathbb{R}^{+} \rightarrow \mathbb{R}^{+}$is the continous function that vanishes on $[0, \varepsilon]$, is equal to 1 on $[2 \varepsilon, \infty)$ and is linear on $[\varepsilon, 2 \varepsilon]$. It is easy to see that $F_{c}(A)$ is a dense subset of $F(A)$, which - unlike $F(A)$-is closed under addition.

We shall use below that whenever $c \in A$ is a strictly positive element of $A$, then $c \otimes 1_{n}$ is a strictly positive element of $M_{n}(A)$. 
Lemma 5.2. Let $c$ be a strictly positive element of a $C^{*}$-algebra $A$, and let $a=\left(a_{i j}\right)$ be a positive element in $M_{n}(A)$. Let $d=\sum_{j=1}^{n} a_{j j} \in A^{+}$be the sum of the diagonal elements of $a$. Then $\langle a\rangle \leq n\langle d\rangle$; and $d$ belongs to $F_{c}(A)$ if a belongs to $F_{c \otimes 1_{n}}\left(M_{n}(A)\right)$.

Proof. Let $\varepsilon>0$. For each $i=1,2, \ldots, n$, let $\left\{e_{k}^{(i)}\right\}_{k=1}^{\infty}$ be an approximate unit for $\overline{a_{i i} A a_{i i}}$, and put $e_{k}=\operatorname{diag}\left(e_{k}^{(1)}, \ldots, e_{k}^{(n)}\right)$. Then $e_{k}^{(i)} \precsim a_{i i} \precsim d$ for all $k$. Also, $\left\{e_{k}\right\}$ is an approximate unit for $\overline{a M_{n}(A) a}$, whence $(a-\varepsilon)_{+} \precsim e_{k}$ for all large enough $k$, cf. Lemma 5.1. We therefore conclude that

$$
\left\langle(a-\varepsilon)_{+}\right\rangle \leq\left\langle e_{k}\right\rangle=\sum_{i=1}^{n}\left\langle e_{k}^{(i)}\right\rangle \leq n\langle d\rangle .
$$

This proves the first claim because $\varepsilon>0$ was arbitrary.

Suppose that $a$ belongs to $F_{c \otimes 1_{n}}\left(M_{n}(A)\right)$. Then there is $\varepsilon>0$ with $g_{\varepsilon}\left(c \otimes 1_{n}\right) a=a$. As $g_{\varepsilon}\left(c \otimes 1_{n}\right)=g_{\varepsilon}(c) \otimes 1_{n}$, this is easily seen to imply that $g_{\varepsilon}(c) a_{i i}=a_{i i}$ for all $i$, hence $a_{i i} \in F_{c}(A)$. Thus $d$ belongs to $F_{c}(A)$.

Lemma 5.3. Let $A$ be a $\sigma$-unital $C^{*}$-algebra, and fix a strictly positive element $c$ in $A$. Suppose that $M_{n}(A)$ is stable for some positive integer $n$. Then for all elements $a, b$ in $F_{c}(A)$ there exists an element $d$ in $F_{c}(A)$ with $a \perp d$ and $b \otimes 1_{n} \precsim d \otimes 1_{n}$.

Proof. Let $a, b \in F_{c}(A)$. Then $a$ and $b$ both belong to $\overline{g_{\delta}(c) A g_{\delta}(c)}$ for some $\delta>0$. Clearly, $a, b \precsim g_{\delta}(c)$ and $g_{\delta}(c) \otimes 1_{n} \in F_{c \otimes 1_{n}}\left(M_{n}(A)\right)$. Using that $M_{n}(A)$ is stable, we find an element $b^{\prime} \in F_{c \otimes 1_{n}}\left(M_{n}(A)\right)$ such that $b^{\prime} \perp g_{\delta}(c) \otimes 1_{n}$ and $g_{\delta}(c) \otimes 1_{n} \precsim b^{\prime}$, cf. [9, Lemma 2.6 (i)]. Let $d \in A$ be the sum of the diagonal elements in $b^{\prime}$. Then $d$ belongs to $F_{c}(A)$ and $b^{\prime} \precsim d \otimes 1_{n}$ by Lemma 5.2. This shows that

$$
b \otimes 1_{n} \precsim g_{\delta}(c) \otimes 1_{n} \precsim b^{\prime} \precsim d \otimes 1_{n} .
$$

Since $b^{\prime} \perp g_{\delta}(c) \otimes 1_{n}$, it follows that $d \perp g_{\delta}(c)$, whence $d \perp a$.

The lemma below is a reformulation of the characterization of stability from [9].

Lemma 5.4. Let $A$ be a $\sigma$-unital $C^{*}$-algebra with a strictly positive element $c$. Then $A$ is stable if and only if for every $\varepsilon>0$ there exists $b \in A^{+}$such that $b \perp(c-\varepsilon)_{+}$and $(c-\varepsilon)_{+} \precsim b$.

Proof. The "only if" part follows from [9, Theorem 2.1]. To prove the "if" part, we verify that condition (b) of [9, Proposition 2.2] is satisfied. To this end, let $a \in F(A)$ and $\varepsilon>0$ be given. Choose $\delta>0$ such that $\left\|a-g_{\delta}(c) a g_{\delta}(c)\right\|<\varepsilon / 2$, put $a^{\prime}=g_{\delta}(c) a g_{\delta}(c)$, and find $d \in A^{+}$such that $(c-\delta)_{+} \perp d$ and $(c-\delta)_{+} \precsim d$. Then $a^{\prime} \perp d$ and $a^{\prime} \precsim g_{\delta}(c) \precsim d$. Hence there exists $t$ in $A$ such that $b^{\prime}:=\left(a^{\prime}-\varepsilon / 2\right)_{+}=t^{*} d t$. Put $c^{\prime}=d^{1 / 2} t t^{*} d^{1 / 2}$. Then $\left\|a-b^{\prime}\right\| \leq \varepsilon, b^{\prime} \perp c^{\prime}$, and $b^{\prime} \sim c^{\prime}$.

Proposition 5.5. Let $A$ be a $\sigma$-unital $C^{*}$-algebra whose Cuntz semigroup $\mathrm{Cu}(A)$ satisfies the Corona Factorization Property for semigroups. Then $A$ is stable if $M_{m}(A)$ is stable for some $m \in \mathbb{N}$. 
Proof. Suppose that $M_{m}(A)$ is stable for some natural number $m$. Let $c$ be a strictly positive element in $A^{+}$, and let $\varepsilon>0$ be given. Choose a decreasing sequence $\left\{\varepsilon_{n}\right\}$ of strictly positive real numbers that converges to zero, and such that $\varepsilon_{1}<\varepsilon$. Let $a_{n}=\left(c-\varepsilon_{n}\right)_{+}$. Since $a_{n}$ is Cuntz equivalent to $g_{\varepsilon_{n}}(c)$ and $\left\{g_{\varepsilon_{n}}(c)\right\}$ is an increasing approximate unit for $\overline{c A c}=A$, it follows from Lemma 5.1 that $\left\{\left\langle a_{n}\right\rangle\right\}$ is a full sequence in $\mathrm{Cu}(A)$.

We use Lemma 5.3 to construct a sequence $d_{1}, d_{2}, d_{3} \ldots$ of positive elements in $F_{c}(A)$ such that $a_{1}, d_{1}, d_{2}, \ldots$ are pairwise orthogonal and $a_{n} \precsim d_{n} \otimes 1_{m}$ for all $n$. Indeed, at stage $n$, since $a_{1}, d_{1}, \ldots, d_{n-1}$ belong to $F_{c}(A)$, so does their sum, and so we can find $d_{n} \in F_{c}(A)$ orthogonal to $a_{1}+d_{1}+\cdots+d_{n-1}$ satisfying $a_{n} \precsim d_{n} \otimes 1_{m}$.

Now, apply the Corona Factorization Property for $\mathrm{Cu}(A)$ to $\left\{\left\langle a_{n}\right\rangle\right\}$ and $\left\{\left\langle d_{n}\right\rangle\right\}$ (that satisfies $\left\langle a_{n}\right\rangle \leq m\left\langle d_{n}\right\rangle$ for all $\left.n\right)$. Thus, for our $\varepsilon>0$, since $\left\langle(c-\varepsilon)_{+}\right\rangle \ll\left\langle\left(c-\varepsilon_{1}\right)_{+}\right\rangle=\left\langle a_{1}\right\rangle$ there is a natural number $k$ such that

$$
\left\langle(c-\varepsilon)_{+}\right\rangle \leq\left\langle d_{1}\right\rangle+\left\langle d_{2}\right\rangle+\cdots+\left\langle d_{k}\right\rangle=\left\langle d_{1}+d_{2}+\cdots+d_{k}\right\rangle,
$$

which implies that $A$ is stable (by virtue of Lemma 5.4).

If $A$ is a non-unital $C^{*}$-algebra, then we shall denote its multiplier algebra by $\mathcal{M}(A)$ and the unit in the multiplicer algebra by 1.

Lemma 5.6. Let $A$ be a $\sigma$-unital stable $C^{*}$-algebra and let a be a positive contraction in $A$. Then $1-a$ is a properly infinite, full, positive element in $\mathcal{M}(A)$.

Proof. It follows from [9, Corollary 4.3] that $\overline{(1-a) A(1-a)}$ is stable. Hence $1-a$ is properly infinite, cf. [10, Proposition 3.7]. We proceed to prove that $1-a$ is full in $\mathcal{M}(A)$.

Take positive functions $f, g:[0,1] \rightarrow[0,1]$ such that $f$ is zero on $[0,1 / 2], f+g=1$, and $g(1)=0$. Then $g(a)$ belongs to $\overline{(1-a) A(1-a)}$. Since $A$ is stable and $\sigma$-unital we can find a positive element $b$ in $A$ such that $b \perp(a-1 / 2)_{+}$and $(a-1 / 2)_{+} \precsim b$. Then $f(a) \perp b$, whence

$$
b=(f(a)+g(a)) b(f(a)+g(a))=g(a) b g(a) \in \overline{(1-a) A(1-a)} .
$$

As $f(a) \precsim(a-1 / 2)_{+} \precsim b$, we see that $f(a)$ belongs to the closed two-sided ideal in $\mathcal{M}(A)$ generated by $1-a$. As $g(a)$ belongs to $\overline{(1-a) \mathcal{M}(A)(1-a)}$, we conclude that the closed two-sided ideal generated by $1-a$ contains $1=f(a)+g(a)$, and hence is equal to $\mathcal{M}(A)$.

Lemma 5.7. Let $A$ be a $\sigma$-unital stable $C^{*}$-algebra and let $T$ be a positive element in $\mathcal{M}(A)$ such that $1 \precsim T$ (or, equivalently, such that $T$ is full and properly infinite). Then $\overline{T A T}$ is stable.

Proof. Put $B=\overline{T A T}$. Since $A$ is $\sigma$-unital, then so is $B$.

There is $\delta>0$ such that $1 \precsim(T-2 \delta)_{+}$, whence $1=R^{*}(T-\delta)_{+} R$ for some element $R$ in $\mathcal{M}(A)$. Put $V=(T-\delta)_{+}^{1 / 2} R$ and put $T^{\prime}=g(T)$, where $g: \mathbb{R}^{+} \rightarrow[0,1]$ is 
a continuous function such that $g(0)=0, g(t)=1$ for $t \geq \delta$, and $g$ is linear on $[0, \delta]$. Then $\overline{T^{\prime} A T^{\prime}}=\overline{T A T}=B$, and $V$ is an isometry whose range projection satisfies $V V^{*} T^{\prime}=V V^{*}$.

To show that $B$ is stable, we use [9], by which it suffices to show that for each $a \in F(A)$ there is $b \in A^{+}$such that $a \perp b$ and $a \sim b$. Take $a \in F(B)$, and let $e$ be a positive contraction in $B$ such that $a e=e a=a$. Put $T_{0}=(1-e) T^{\prime}(1-e)$, and note that $\overline{T_{0} A T_{0}} \subseteq B$. Now,

$$
V^{*} T_{0} V=V^{*} T^{\prime} V-V^{*}\left(e T^{\prime}+T^{\prime} e-e T^{\prime} e\right) V=1-c,
$$

with $c=V^{*}\left(e T^{\prime}+T^{\prime} e-e T^{\prime} e\right) V \in A$. As $V^{*} T_{0} V$ is a positive contraction, the element $c$ is also a positive contraction. We can therefore use Lemma 5.6 to conclude that $V^{*} T_{0} V$ is properly infinite and full. As $V^{*} T_{0} V \precsim T_{0}$ we also have that $T_{0}$ is properly infinite and full. This again entails that $1 \precsim T_{0}$, and so there is an isometry $W$ in $\mathcal{M}(A)$ whose range projection, $W W^{*}$, belongs to $\overline{T_{0} \mathcal{M}(A) T_{0}}$. In particular, $W W^{*} \perp a$. Put $b=W a W^{*}$. Then $b$ is a positive element in $B, b \perp a$, and $b \sim a$ as desired.

Let $A$ be a stable $C^{*}$-algebra. Then there exists a sequence $\left\{S_{n}\right\}$ of isometries in $\mathcal{M}(A)$ with orthogonal range projections and such that $\sum_{n=1}^{\infty} S_{n} S_{n}^{*}=1$ (the sum being convergent in the strict topology). Let $\left\{a_{n}\right\}$ be any bounded sequence of elements in $A$ (or in $\mathcal{M}(A)$ ). Then $\sum_{n=1}^{\infty} S_{n} a_{n} S_{n}^{*}$ is strictly convergent to an element in $\mathcal{M}(A)$. We shall denote this element by $\bigoplus_{n=1}^{\infty} a_{n}$. If $\left\{T_{n}\right\}$ is another sequence of isometries in $\mathcal{M}(A)$ with range projections adding up to 1 in the strict topology, then $\sum_{n=1}^{\infty} T_{n} S_{n}^{*}$ is strictly convergent to a unitary $U$ in $\mathcal{M}(A)$ and $U\left(\sum_{n=1}^{\infty} S_{n} a_{n} S_{n}^{*}\right) U^{*}=\sum_{n=1}^{\infty} T_{n} a_{n} T_{n}^{*}$. This shows that the element $\bigoplus_{n=1}^{\infty} a_{n}$ is independent on the choice of the sequence $\left\{S_{n}\right\}$ of isometries, up to unitary equivalence.

Lemma 5.8. Let $A$ be a stable $\sigma$-unital $C^{*}$-algebra which satisfies the Corona Factorization Property. Let $T$ be a full, positive element in $\mathcal{M}(A)$. Then a $T$ for every positive element a in $A$.

Proof. Put $B=\overline{T A T}$. Then $B$ is a full hereditary sub- $C^{*}$-algebra of $A$ because $T$ is full in $\mathcal{M}(A)$.

Again using that $T$ is a full element in the properly infinite $C^{*}$-algebra $\mathcal{M}(A)$, there is a positive integer $n$ such that $T \otimes 1_{n}$ is properly infinite. As,

$$
M_{n}(B)=\overline{\left(T \otimes 1_{n}\right) M_{n}(A)\left(T \otimes 1_{n}\right)},
$$

we conclude from Lemma 5.7 that $M_{n}(B)$ is stable. Because $A$ is assumed to satisfy the Corona Factorization Property, we can now conclude from [12, Theorem 4.2] that $B$ is stable.

Let $a$ be a positive element in $A$ and let $\varepsilon>0$ be given. As $B$ is full in $A$ we can find a positive integer $n$, positive elements $b_{1}, \ldots, b_{n}$ in $B$, and elements $x_{1}, \ldots, x_{n}$ in $A$ such that $(a-\varepsilon)_{+}=\sum_{j=1}^{n} x_{j}^{*} b_{j} x_{j}$. Because $B$ is stable there are isometries $S_{1}, \ldots, S_{n}$ 
in $\mathcal{M}(B)$ with orthogonal range projections. We now get

$$
(a-\varepsilon)_{+} \precsim b_{1} \oplus b_{2} \oplus \cdots \oplus b_{n} \approx S_{1} b_{1} S_{1}^{*}+S_{2} b_{2} S_{2}^{*}+\cdots+S_{n} b_{n} S_{n}^{*} \precsim T .
$$

As this holds for all $\varepsilon>0$, we have $a \precsim T$ as desired.

The lemma below is similar to [17, Corollary 2.7], but we do not assume below that $A$ is unital. If $a$ and $b$ are positive elements in a $C^{*}$-algebra and if $m$ is a positive integer, then we shall write $a{ }_{m} b$ to denote that $a \precsim b \otimes 1_{m}$.

Lemma 5.9. Let $A$ be a $\sigma$-unital stable $C^{*}$-algebra, and let $c$ be a strictly positive contraction in $A$.

Let $\left\{a_{n}\right\}$ be a bounded sequence of positive elements in $A$. Then $\bigoplus_{n=1}^{\infty} a_{n}$ defines a full element in $\mathcal{M}(A)$ if there exist $\delta>0$ and a positive integer $m$ such that for every $\varepsilon>0$ and for every positive integer $k$ there is an integer $\ell>k$ such that

$$
(c-\varepsilon)_{+} \precsim m\left(a_{k}-\delta\right)_{+} \oplus\left(a_{k+1}-\delta\right)_{+} \oplus \cdots \oplus\left(a_{\ell}-\delta\right)_{+} \cdot
$$

Proof. We show that $1 \precsim_{m} \bigoplus_{n=1}^{\infty} a_{n}$, which of course will imply that $\bigoplus_{n=1}^{\infty} a_{n}$ is full. By assumption we can find integers $1=k_{1}<k_{2}<k_{3}<\cdots$ such that

$$
\left(c-\frac{1}{n}\right)_{+} \precsim m\left(a_{k_{n}}-\delta\right)_{+} \oplus\left(a_{k_{n}+1}-\delta\right)_{+} \oplus \cdots \oplus\left(a_{k_{n+1}-1}-\delta\right)_{+}
$$

for all $n$.

Choose isometries $T_{1}, T_{2}, \ldots, T_{m}$ in $\mathcal{M}(A)$ with range projections adding up to 1. Then we can identify $\left(\bigoplus_{n=1}^{\infty} a_{n}\right) \otimes 1_{m}$ with

$$
\sum_{j=1}^{m} T_{j}\left(\bigoplus_{n=1}^{\infty} a_{n}\right) T_{j}^{*}=\sum_{n=1}^{\infty} \sum_{j=1}^{m} T_{j} S_{n} a_{n} S_{n}^{*} T_{j}^{*} \sim \sum_{n=1}^{\infty} S_{n}\left(\sum_{j=1}^{m} T_{j} a_{n} T_{j}^{*}\right) S_{n}^{*}
$$

(We have here used that the range projections of the two families of isometries, $\left\{S_{n} T_{j}\right\}$ and $\left\{T_{j} S_{n}\right\}$, sum to 1 in the strict topology.) Put

$$
b_{n}=\sum_{k=k_{n}}^{k_{n+1}-1} S_{k}\left(\sum_{j=1}^{m} T_{j} a_{n} T_{j}^{*}\right) S_{k}^{*} \sim\left(a_{k_{n}} \oplus a_{k_{n}+1} \oplus \cdots \oplus a_{k_{n+1}-1}\right) \otimes 1_{m} .
$$

Then $\left(\bigoplus_{n=1}^{\infty} a_{n}\right) \otimes 1_{m} \sim \sum_{n=1}^{\infty} b_{n}$, the latter sum is strictly convergent, and $\left(c-\frac{1}{n}\right)_{+} \precsim$ $\left(b_{n}-\delta\right)_{+}$for all $n$. We must show that $1 \precsim \sum_{n=1}^{\infty} b_{n}$.

Choose a strictly decreasing sequence $\left\{\delta_{n}\right\}$ of positive real numbers such that $\delta_{2}=1$ and $\delta_{n+2}>1 / n$ for all $n$. Define $g_{n}:[0,1] \rightarrow[0,1]$ to be the continuous function which is zero on $\left[0, \delta_{n+2}\right] \cup\left[\delta_{n}, 1\right]$ (note that $\left[\delta_{1}, 1\right]=\emptyset$ ), $g_{n}\left(\delta_{n+1}\right)=1$, and $g_{n}$ is linear on $\left[\delta_{n+2}, \delta_{n+1}\right]$ and on $\left[\delta_{n+1}, \delta_{n}\right]$. Then $1=\sum_{n=1}^{\infty} g_{n}(c)$ and the sum is strictly convergent. Moreover, since $\delta_{n+2}>1 / n$, we have $g_{n}(c)=x_{n}^{*}\left(b_{n}-\delta\right)_{+} x_{n}$ for some element $x_{n}$ in $A$. Let $h:[0,1] \rightarrow \mathbb{R}^{+}$be the continuous function which satisfies $h(0)=0, h(t)=t^{-1 / 2}$ for $t \geq \delta$, and $h$ is linear on $[0, \delta]$. Put $y_{n}=h\left(b_{n}\right)\left(b_{n}-\delta\right)_{+}^{1 / 2} x_{n}$. Then $\left\|y_{n}\right\| \leq \delta^{-1 / 2}$ (because $\left\|\left(b_{n}-\delta\right)_{+}^{1 / 2} x_{n}\right\|=\left\|g_{n}(c)\right\|^{1 / 2}=1$ and $\left\|h\left(b_{n}\right)\right\| \leq \delta^{-1 / 2}$ ), and $y_{n}^{*} b_{n} y_{n}=g_{n}(c)$. 
Notice that $y_{n}$ belongs to the set $\overline{b_{n} A g_{n}(c)}$. Put $Y=\sum_{n=1}^{\infty} y_{n} \in \mathcal{M}(A)$ (the sum is strictly convergent). Then,

$$
Y^{*}\left(\sum_{n=1}^{\infty} b_{n}\right) Y=\sum_{n=1}^{\infty} Y^{*} b_{n} Y=\sum_{n=1}^{\infty} y_{n}^{*} b_{n} y_{n}=\sum_{n=1}^{\infty} g_{n}(c)=1,
$$

which shows that $1 \precsim \sum_{n=1}^{\infty} b_{n}$.

Lemma 5.10. Let $A$ be a stable $\sigma$-unital $C^{*}$-algebra which satisfies the Corona Factorization Property. Let $a_{1}, a_{2}, \ldots, b_{1}, b_{2}, \ldots$ be positive elements in $A$, and let $m$ be $a$ positive integer such that $a_{1} \precsim a_{2} \precsim a_{3} \precsim \cdots$, such that the set $\left\{a_{n}\right\}$ is full in $A$, and such that $a_{n} \precsim b_{n} \otimes 1_{m}$ for all $n$. It follows that for each $\eta>0$ there is a natural number $k$ such that

$$
\left(a_{1}-\eta\right)_{+} \precsim b_{1} \oplus b_{2} \oplus \cdots \oplus b_{k} .
$$

Proof. We note first that we can choose $\delta_{n}>0$ such that

$$
\left(a_{1}-\delta_{1}\right)_{+} \precsim\left(a_{2}-\delta_{2}\right)_{+} \precsim\left(a_{3}-\delta_{3}\right) \precsim \cdots,
$$

and such that $\left\{\left(a_{n}-\delta_{n}\right)_{+}\right\}_{n=1}^{\infty}$ is full in $A$. (Let us prove this fact: As $a_{j} \precsim a_{n}$ whenever $1 \leq j<n$ there is $\eta_{n}>0$ such that $\left(a_{j}-1 / n\right)_{+} \precsim\left(a_{n}-\eta_{n}\right)_{+}$for $j=$ $1,2, \ldots, n-1$. We choose now $\delta_{n}$ inductively such that $0<\delta_{n} \leq \eta_{n}$ and such that $\left(a_{n-1}-\delta_{n-1}\right)_{+} \precsim\left(a_{n}-\delta_{n}\right)_{+}$. For $n=1$ we can take $\delta_{1}=\eta_{1}$. For $n \geq 2$, since $a_{n-1} \precsim a_{n}$, there is $\delta_{n} \in\left(0, \eta_{n}\right]$ such that $\left(a_{n-1}-\delta_{n-1}\right)_{+} \precsim\left(a_{n}-\delta_{n}\right)_{+}$. To see that the sequence $\left\{\left(a_{n}-\delta_{n}\right)_{+}\right\}_{n=1}^{\infty}$ is full in $A$, let $I$ be the closed two-sided ideal generated by this sequence. Since $\left(a_{j}-1 / n\right)_{+} \precsim\left(a_{n}-\eta_{n}\right)_{+} \precsim\left(a_{n}-\delta_{n}\right)_{+} \in I$ whenever $1 \leq j<n$, we see that $\left(a_{j}-1 / n\right)_{+}$belongs to $I$ whenever $n>j$. It follows that $a_{j}$ belongs to $I$ for all $j$, whence $I=A$, because the sequence $\left\{a_{n}\right\}$ was assumed to be full.)

Next we choose $\delta_{n}^{\prime}>0$ such that $\left(a_{n}-\delta_{n}\right)_{+} \precsim_{m}\left(b_{n}-\delta_{n}^{\prime}\right)_{+}$for all $n$. Let $g_{n}:[0,1] \rightarrow$ $[0,1]$ be the continuous function given by $g_{n}(0)=0, g_{n}(t)=1$ for $t \geq \delta_{n}^{\prime}$, and $g_{n}$ is linear on $\left[0, \delta_{n}^{\prime}\right]$. Put $b_{n}^{\prime}=g_{n}\left(b_{n}\right)$. Then $b_{n}$ is Cuntz equivalent to $b_{n}^{\prime}$, and $\left(b_{n}-\delta_{n}^{\prime}\right)_{+} \precsim$ $\left(b_{n}^{\prime}-1 / 2\right)_{+}$.

We claim that $T:=\bigoplus_{n=1}^{\infty} b_{n}^{\prime}$ is full in $\mathcal{M}(A)$. To this end, take a strictly positive contraction $c$ in $A$. Let $k \in \mathbb{N}$ and $\varepsilon>0$ be given. Note that the tail $\left\{\left(a_{n}-\delta_{n}\right)_{+}\right\}_{n=k}^{\infty}$ is full in $A$ (because the sequence $\left\{\left(a_{n}-\delta_{n}\right)_{+}\right\}_{n=1}^{\infty}$ is Cuntz increasing). It follows that $c$ belongs to the closed two-sided ideal generated by $\left\{\left(a_{n}-\delta_{n}\right)_{+}\right\}_{n=k}^{\infty}$, whence $(c-\varepsilon)_{+}$ belongs to the algebraic ideal generated by this sequence, and hence to the algebraic ideal generated by $\left\{\left(a_{n}-\delta_{n}\right)_{+}\right\}_{n=k}^{k^{\prime}}$ for some $k^{\prime}>k$. This entails that

$$
(c-\varepsilon)_{+} \precsim_{p}\left(a_{k}-\delta_{k}\right)_{+} \oplus\left(a_{k+1}-\delta_{k+1}\right)_{+} \oplus \cdots \oplus\left(a_{k^{\prime}}-\delta_{k^{\prime}}\right)_{+},
$$

for some positive integer $p$. Using again the sequence $\left\{\left(a_{n}-\delta_{n}\right)_{+}\right\}_{n=1}^{\infty}$ is Cuntz increasing, we get that

$$
\begin{aligned}
(c-\varepsilon)_{+} & \precsim\left(a_{k}-\delta_{k}\right)_{+} \oplus\left(a_{k+1}-\delta_{k+1}\right)_{+} \oplus \cdots \oplus\left(a_{\ell}-\delta_{\ell}\right)_{+} \\
& \precsim m\left(b_{k}^{\prime}-1 / 2\right)_{+} \oplus\left(b_{k+1}^{\prime}-1 / 2\right)_{+} \oplus \cdots \oplus\left(b_{\ell}^{\prime}-1 / 2\right)_{+},
\end{aligned}
$$


when $\ell \geq k+p\left(k^{\prime}-k+1\right)$. Lemma 5.9 now yields that $T$ is full in $\mathcal{M}(A)$.

Since $A$ is assumed to have the Corona Factorization Property we can use Lemma 5.8 to conclude that $a_{1} \precsim T$. Hence $\left(a_{1}-\eta / 2\right)_{+}=R^{*} T R$ for some $R$ in $\mathcal{M}(A)$. Take a positive contraction $e$ in $A$ such that $e\left(a_{1}-\eta / 2\right)_{+}=\left(a_{1}-\eta / 2\right)_{+}=\left(a_{1}-\eta / 2\right)_{+} e$. Put $r=R e \in A$. As $\bigoplus_{n=1}^{k} b_{n}^{\prime} \rightarrow T$ in the strict topology as $k \rightarrow \infty$, it follows that $r^{*}\left(\bigoplus_{n=1}^{k} b_{n}^{\prime}\right) r \rightarrow r^{*} \operatorname{Tr}=\left(a_{1}-\eta / 2\right)_{+}$in the norm topology (on $A$ ) as $k \rightarrow \infty$. Take $k$ such that

$$
\left\|r^{*}\left(b_{1}^{\prime} \oplus b_{2}^{\prime} \oplus \cdots \oplus b_{k}^{\prime}\right) r-\left(a_{1}-\eta / 2\right)_{+}\right\|<\eta / 2 .
$$

Then

$$
\left(a_{1}-\eta\right)_{+} \precsim r^{*}\left(b_{1}^{\prime} \oplus b_{2}^{\prime} \oplus \cdots \oplus b_{k}^{\prime}\right) r \precsim b_{1}^{\prime} \oplus b_{2}^{\prime} \oplus \cdots \oplus b_{k}^{\prime} \approx b_{1} \oplus b_{2} \oplus \cdots \oplus b_{k}
$$

as desired.

Theorem 5.11. Let $A$ be a $\sigma$-unital $C^{*}$-algebra. Then $A$ has the Corona Factorization Property if and only if its Cuntz semigroup, $\mathrm{Cu}(A)$, has the Corona Factorization Property (for semigroups).

Proof. Assume first that $A$ has the Corona Factorization Property. Let $\left\{x_{n}\right\}$ be a full sequence in $\mathrm{Cu}(A)$, let $\left\{y_{n}\right\}$ be another sequence in $\mathrm{Cu}(A)$, let $x^{\prime} \in \mathrm{Cu}(A)$, and let $m \in \mathbb{N}$ be such that $x_{n} \leq m y_{n}$ for all $n$ and $x^{\prime} \ll x_{1}$. Take positive elements $a_{n}$ and $b_{n}$ in $A \otimes \mathcal{K}$ such that $x_{n}=\left\langle a_{n}\right\rangle$ and $y_{n}=\left\langle b_{n}\right\rangle$, and take $\eta>0$ such that $x^{\prime} \leq\left\langle\left(a_{1}-\eta\right)_{+}\right\rangle$. Then $\left\{a_{n}\right\}$ is full in $A \otimes \mathcal{K}, a_{1} \precsim a_{2} \precsim \cdots$, and $a_{n} \precsim b_{n} \otimes 1_{m}$ for all $n$. Hence, by Lemma 5.10, we get that

$$
\left(a_{1}-\eta\right)_{+} \precsim b_{1} \oplus b_{2} \oplus \cdots \oplus b_{k}
$$

for some $k$. Thus

$$
x^{\prime} \leq\left\langle\left(a_{1}-\eta\right)_{+}\right\rangle \leq\left\langle b_{1}\right\rangle+\left\langle b_{2}\right\rangle+\cdots+\left\langle b_{k}\right\rangle=y_{1}+y_{2}+\cdots+y_{k} .
$$

This shows that $\mathrm{Cu}(A)$ has the Corona Factorization Property.

For the converse direction it is shown in [12, Theorem 4.2] that $A$ has the Corona Factorization Property if and only if for every full hereditary sub- $C^{*}$-algebra $B$ of $A$ one has that $B$ is stable if some matrix algebra over $B$ is stable. $\mathrm{As} \mathrm{Cu}(B) \cong \mathrm{Cu}(A)$ for all such $B$, this follows from Proposition 5.5.

Corollary 5.12. Let $A$ be a separable, unital $C^{*}$-algebra with finite decomposition rank. Then $A$ has the Corona Factorization Property.

Proof. Combine Theorem 5.11 above with Corollary 3.3.

The corollary above extends the result of Pimsner, Popa and Voiculescu, [15], and Kucerovsky and $\mathrm{Ng}$, [13], that the $C^{*}$-algebra $C(X) \otimes \mathcal{K}$ is absorbing, or equivalently, that it satisfies the Corona Factorization Property, when $X$ has finite covering dimension (as the decomposition rank of $C(X) \otimes \mathcal{K}$ coincides with the covering dimension of the space $X)$. 
We end this paper by describing for which $C^{*}$-algebras the Cuntz semigroup has the strong Corona Factorization Property.

Theorem 5.13. Let $A$ be a separable $C^{*}$-algebra. Then $\mathrm{Cu}(A)$ has the strong Corona Factorization Property if and only if every ideal in $A$ has the Corona Factorization Property.

Proof. Assume that $\mathrm{Cu}(A)$ has the strong Corona Factorization Property, and let $I$ be a closed two-sided ideal in $A$. Then $\mathrm{Cu}(I)$ is an ideal in $\mathrm{Cu}(A)$. As the strong Corona Factorization Property trivially passes to ideals, we conclude that $\mathrm{Cu}(I)$ satisfies the (strong) Corona Factorization Property. It therefore follows from Theorem 5.11 that $I$ has the Corona Factorization Property (for $C^{*}$-algebras).

Suppose now that all ideals in $A$ have the Corona Factorization Property. To show that $\mathrm{Cu}(A)$ has the strong Corona Factorization Property, it suffices to show that whenever $a, b_{1}, b_{2}, \ldots$ are positive elements in $A \otimes \mathcal{K}, \varepsilon>0$, and $m$ is a positive integer such that $a \precsim b_{n} \otimes 1_{m}$, then $(a-\varepsilon)_{+} \precsim b_{1} \oplus b_{2} \oplus \cdots \oplus b_{k}$ for some positive integer $k$. There are elements $t_{n} \in A \otimes \mathcal{K}$ such that $t_{n}^{*}\left(b_{n} \otimes 1_{m}\right) t_{n}=(a-\varepsilon / 3 n)_{+}$.

Let $I$ be the closed two-sided ideal in $A \otimes \mathcal{K}$ generated by $a$. Then $a$ is full in $I$, and $\langle a\rangle$ is full in $\mathrm{Cu}(I)$; however, the elements $b_{n}$ may not belong to $I$. To fix this problem, take a quasi-central increasing approximate unit $\left\{e_{k}\right\}_{k=1}^{\infty}$ for $I$ consisting of positive contractions. For each $n$ find $k$ such that

$$
\left\|t_{n}^{*}\left(e_{k} \otimes 1_{m}\right)\left(b_{n} \otimes 1_{m}\right)\left(e_{k} \otimes 1_{m}\right) t_{n}-(a-\varepsilon / 3 n)_{+}\right\|<\varepsilon / 3 n,
$$

and put $a_{n}=(a-2 \varepsilon / 3 n)_{+}$and $c_{n}=e_{k} b_{n} e_{k}$. Then $c_{n}$ belongs to $I, c_{n} \precsim b_{n}, a_{n} \precsim c_{n} \otimes 1_{m}$ (relatively to $A$, and hence also relatively to $I),(a-\varepsilon)_{+}=\left(a_{1}-\varepsilon / 3\right)_{+}$, and $\left\{\left\langle a_{n}\right\rangle\right\}$ is a full sequence in $\mathrm{Cu}(I)$. Since $\mathrm{Cu}(I)$ is assumed to satisfy the Corona Factorization Property we conclude that

$$
(a-\varepsilon)_{+} \precsim c_{1} \oplus c_{2} \oplus \cdots \oplus c_{k} \precsim b_{1} \oplus b_{2} \oplus \cdots \oplus b_{k}
$$

for some $k$ as desired.

\section{ACKNOWLEDGEMENTS}

The first and second named authors were partially supported by a MEC-DGESIC grant (Spain) through Project MTM2008-0621-C02-01/MTM, and by the Comissionat per Universitats i Recerca de la Generalitat de Catalunya. The third named author was supported by a grant from the Danish Natural Science Research Council (FNU). Part of this research was carried out during visits of the first and third named authors to UAB (Barcelona), of the second named author to SDU (Odense), and of the two first mentioned authors to Copenhagen. We wish to thank all parties involved for the hospitality extended to us. 


\section{REFERENCES}

[1] Antoine, R., Bosa, J. And Perera, F., Completions of monoids with applications to the Cuntz semigroup. Preprint.

[2] Blackadar, B. And Handelman, D., Dimension functions and traces on $C^{*}$-algebras, J. Funct. Anal. 45 (1982), no. 3, 297-340.

[3] Bosa, J. AND Rørdam, M., In preparation.

[4] Coward, K.T., Elliott, G.A., Ivanescu, C., The Cuntz semigroup as an invariant for $C^{*}-$ algebras, J. Reine Angew. Math. 623 (2008), 161-193.

[5] Cuntz, J., Dimension Functions on Simple $C^{*}$-algebras, Math. Ann. 233 (1978), 145-153.

[6] Elliott, G.A. And Kucerovsky, D., An abstract Voiculescu-Brown-Douglas-Fillmore absorption theorem. Pacific J. Math. 198 (2001), no. 2, 385-409.

[7] Goodearl, K. R. And Handelman, D., Rank functions and $K_{0}$ of regular rings, J. Pure Appl. Algebra, 7 (1976), 195-216.

[8] Hirshberg, I., Rørdam, M. And Winter, W., $C_{0}(X)$-algebras, stability and strongly selfabsorbing $C^{*}$-algebras, Math. Ann. 339 (2007), no. 3, 695-732.

[9] Huelmborg, J. And Rørdam, M., On stability of $C^{*}$-algebras, J. Funct. Anal. 155 (1998), no. $1,153-170$.

[10] Kirchberg, E. And Rørdam, M., Non-simple purely infinite $C^{*}$-algebras, Amer. J. Math. 122 (2000), no. 3, 637-666.

[11] Kirchberg, E. AND Winter, W., Covering dimension and quasidiagonality, International J. Math. 15 (2004), 63-85.

[12] Kucerovsky, D. And NG, P. W., S-regularity and the corona factorization property, Math. Scand. 99 (2006), no. 2, 204-216.

[13] Kucerovsky, D. And NG, P. W., Decomposition rank and absorbing extensions of type I algebras, J. Funct. Anal. 221 (2005), no. 1, 25-36.

[14] Ortega, E., Perera, F. And Rørdam, M., The Corona Factorization Property and Refinement Monoids. Preprint, 2009.

[15] Pimsner, M., Popa, S. and Voiculescu, D., Homogeneous $C^{*}$-extensions of $C(X) \otimes K(H)$. I, J. Operator Theory 1 (1979), no. 1, 55-108.

[16] Robert, L., Nuclear dimension and $n$-comparison. Preprint, 2009.

[17] Rørdam, M., Ideals in the Multiplier Algebra of a Stable $C^{*}$-algebra, J. Operator Theory 25 (1991), no. 2, 283-298.

[18] Rørdam, M., On the Structure of Simple $C^{*}$-algebras Tensored with a UHF-Algebra, II, J. Funct. Anal. 107 (1992), 255-269.

[19] RøRdam, M., Stability of $C^{*}$-algebras is not a stable property, Doc. Math. 2 (1997), 375-386

[20] RøRDAm, M., On sums of finite projections, in "Operator algebras and operator theory (Shanghai, 1997)" Amer. Math. Soc., Providence, RI. (1998), 327-340.

[21] Rørdam, M., A simple $C^{*}$-algebra with a finite and an infinite projection, Acta Math. 191 (2003), no. 1, 109-142.

[22] Rørdam, M., The Stable and the Real Rank of $\mathcal{Z}$-absorbing $C^{*}$-algebras, International J. Math. 15 (2004), no. 10, 1065-1084.

[23] Toms, A. And Winter, W., The Elliott Conjecture for Villadsen Algebras of first type, Preprint. 
Department of Mathematics and Computer Science, University of Southern Denmark, Campusvej 55, DK-5230, Odense M, Denmark

E-mail address: ortega@imada.sdu.dk

Departament de Matemàtiques, Universitat Autònoma de Barcelona, 08193 BelLATERra, BARCELONA, SPAIN

E-mail address: perera@mat.uab.cat

Department of Mathematical Sciences, University of Copenhagen, Universitetsparken 5, DK-2100, Copenhagen $\varnothing$, Denmark

E-mail address: rordam@math.ku.dk 